\title{
UNIFORM STABILIZATION OF A VISCOUS NUMERICAL APPROXIMATION FOR A LOCALLY DAMPED WAVE EQUATION*
}

\author{
Arnaud MÜNCH ${ }^{1}$ And Ademir Fernando Pazoto ${ }^{2}$
}

\begin{abstract}
This work is devoted to the analysis of a viscous finite-difference space semi-discretization of a locally damped wave equation in a regular 2-D domain. The damping term is supported in a suitable subset of the domain, so that the energy of solutions of the damped continuous wave equation decays exponentially to zero as time goes to infinity. Using discrete multiplier techniques, we prove that adding a suitable vanishing numerical viscosity term leads to a uniform (with respect to the mesh size) exponential decay of the energy for the solutions of the numerical scheme. The numerical viscosity term damps out the high frequency numerical spurious oscillations while the convergence of the scheme towards the original damped wave equation is kept, which guarantees that the low frequencies are damped correctly. Numerical experiments are presented and confirm these theoretical results. These results extend those by Tcheugoué-Tébou and Zuazua [Numer. Math. 95, 563-598 (2003)] where the 1-D case was addressed as well the square domain in 2-D. The methods and results in this paper extend to smooth domains in any space dimension.
\end{abstract}

Mathematics Subject Classification. 65M06.

Received June 4, 2004. Revised August 10, 2005.

\section{INTRODUCTION AND SETTING OF THE PROBLEM}

Let us consider the following damped wave equation defined on $\Omega \times[0, \infty), \Omega$ being a bounded domain in $\mathbb{R}^{2}$ of class $C^{2}$ :

$$
\left\{\begin{array}{l}
y^{\prime \prime}-\Delta y+a(\boldsymbol{x}) y^{\prime}=0 \text { in } \Omega \times(0, \infty), \\
y=0 \text { on } \Gamma \equiv \partial \Omega \times(0, \infty), \\
y(\boldsymbol{x}, 0)=y_{0}(\boldsymbol{x}) \text { in } \Omega, \\
y^{\prime}(\boldsymbol{x}, 0)=y_{1}(\boldsymbol{x}) \text { in } \Omega,
\end{array}\right.
$$

where the symbol ' designates the partial differentiation with respect to time. The damping potential $a=a(\boldsymbol{x})$ is assumed to be a bounded nonnegative function defined on $\Omega$. In addition, we suppose that there exists a

\footnotetext{
Keywords and phrases. Wave equation, stabilization, finite difference, viscous terms.

* This work was done while the authors were visiting the Department of Mathematics of the Universidad Autónoma de Madrid. The first author was supported by Grants HPRN-CT-2002-00284 of the European program New materials, adaptive systems and their nonlinearities: modelling, control and numerical simulation. The second author was supported by Grants SB2001-0158 of the MECD (Spain) and BFM 2002-03345 of the MCYT (Spain).

${ }^{1}$ Laboratoire de Mathématiques de Besançon, UMR CNRS 6623, UFR de Sciences et Techniques, Université de Franche-Comté, 16, route de Gray 25030, Besançon Cedex, France; arnaud.munch@univ-fcomte.fr

${ }^{2}$ Instituto de Matemática, Universidade Federal do Rio de Janeiro, 21940-970, Rio de Janeiro, Brasil; ademir@acd.ufrj.br
} 
positive constant $\alpha_{0}$ such that

$$
a(\boldsymbol{x}) \geq \alpha_{0} \text { a.e. } \boldsymbol{x} \in \omega \text { where } \omega \text { is a nonempty open subset of } \Omega \text {. }
$$

System (1) is well-posed in the space $H_{0}^{1}(\Omega) \times L^{2}(\Omega)$. Indeed, given $\left\{y_{0}, y_{1}\right\} \in H_{0}^{1}(\Omega) \times L^{2}(\Omega)$, there exists a unique solution of (1) with (see [16])

$$
y \in \mathcal{C}\left([0, \infty) ; H_{0}^{1}(\Omega)\right) \cap \mathcal{C}^{1}\left([0, \infty) ; L^{2}(\Omega)\right) .
$$

The energy of the system (1) is given by

$$
E(t)=\frac{1}{2} \int_{\Omega}\left\{\left|y^{\prime}(\boldsymbol{x}, t)\right|^{2}+|\nabla y(\boldsymbol{x}, t)|^{2}\right\} \mathrm{d} x, \quad \forall t \geq 0 .
$$

Moreover, the following dissipation law holds

$$
E^{\prime}(t)=-\int_{\Omega} a(\boldsymbol{x})\left|y^{\prime}(\boldsymbol{x}, t)\right|^{2} \mathrm{~d} x, \quad \forall t \geq 0 .
$$

Consequently, $E$ is a non increasing function of the time variable $t$ and therefore a rate of decay is expected.

When $a(\boldsymbol{x}) \geq \alpha_{0}>0$ a.e. in $\Omega$, it is straightforward to see that the energy decays uniformly exponentially as $t$ goes to infinity: there exist two positive constants $C$ and $\alpha$ such that

$$
E(t) \leq C \mathrm{e}^{-\alpha t} E(0), \quad \forall t \geq 0 .
$$

The problem of stabilization when the damping is effective only on a subset of $\Omega$ is much more subtle. This problem has been extensively investigated in the context of wave equations and there is a large literature on the subject. For instance, Dafermos [6], Haraux [8] and Slemrod [22] used La Salle's invariance principle to obtain asymptotic stability properties. More recently, assuming that the dissipation is effective in a suitable subset of the domain where the equation holds, decay rates have been obtained (see for instance Nakao [18] and Zuazua [25]). In particular, when $\omega$ is a neighborhood of the subset of the boundary $\partial \Omega$ as follows

$$
\omega \text { is the intersection of } \Omega \text { and a neighborhood of } \Gamma_{0} \text {, }
$$

where

$$
\Gamma_{0}=\{\boldsymbol{x} \in \partial \Omega ; \boldsymbol{x} \cdot \boldsymbol{\nu}>0\},
$$

and $\boldsymbol{\nu}$ the unit normal pointing into the exterior of $\Omega$ then, the uniform exponential decay (6) holds [9,14, 23, 25].

In view of (5), the problem of the exponential decay of $E$ can be stated in the following equivalent form: is it possible to find $T>0$ and $C>0$ such that

$$
E(0) \leq C \int_{0}^{T} \int_{\Omega} a(\boldsymbol{x})\left|y^{\prime}(\boldsymbol{x}, t)\right|^{2} \mathrm{~d} x \mathrm{~d} t
$$

holds for every finite energy solution of (1)? More precisely, (9) combined with (5) and the semigroup property, allows to obtain the exponential decay property (6). However, we emphasize that, in the general multidimensional setting, inequalities of the form (9) are valid if and only if the subset $\omega$ where the damping is effective satisfies a suitable Geometric Control Condition (GCC) (see [3]). Roughly speaking, the GCC requires that every ray of geometric optics reaches the region in which the damping mechanism is effective in a uniform time, a property that holds in the particular case where $\omega$ is given by (7)-(8). 
We address in this work the numerical approximation of this problem which is by now well-know to be extremely sensitive: for instance, the most classical finite-difference semi-discrete numerical approximation of the first equation of (1)

$$
y_{j, k}^{\prime \prime}(t)-\frac{y_{j+1, k}(t)-2 y_{j, k}(t)+y_{j-1, k}(t)}{h_{1}^{2}}-\frac{y_{j, k+1}(t)-2 y_{j, k}(t)+y_{j, k-1}(t)}{h_{2}^{2}}+a_{j, k} y_{j, k}^{\prime}(t)=0
$$

leads to a semi-discrete observability equality similar to (9) where the constant $C_{h_{1}, h_{2}}$ depending now on the mesh size $h_{1}, h_{2}$ (defined in details in Sect. 2) may blows-up as $h_{1}, h_{2}$ go to zero, depending mainly on the regularity of the initial conditions. Therefore, the exponential decay rate $\alpha_{h_{1}, h_{2}}$ of the discretized energy is not uniform with respect to the discretization parameters. This numerical pathology is independent of the consistence and stability properties of the scheme. This is due to the fact that the interaction of waves with a numerical mesh produces dispersion phenomena and spurious high frequency oscillations. Because of this nonphysical interaction the velocity of propagation of such numerical waves, the so called group velocity may converge to zero when the wavelength of solutions is of order of the mesh size $O\left(h_{1}, h_{2}\right)$ leading to a time of observability of order $1 / O\left(h_{1}, h_{2}\right)$. Such a pathology was suggested by numerical simulations in a preliminary work in [2]. According to the close links between stabilization and exact controllability [12], same bad behaviors appear for the numerical schemes devoted to exact controllability problems (we refer the reader to [28] and the references therein for an extended survey on this issue).

The problem of numerical approximation and stabilization has been addressed in [24] in 1-D and 2-D in the interval and the unit square domain, respectively. It was shown that an estimate similar to (9) is valid for the "finite-difference space semi-discretization" of (1), which is uniform with respect to the mesh size, provided a suitable viscosity term is added. More precisely, the authors replaced equation (10) by the following one:

$$
\begin{aligned}
y_{j, k}^{\prime \prime}(t) & -\frac{y_{j+1, k}(t)-2 y_{j, k}(t)+y_{j-1, k}(t)}{h_{1}^{2}}-\frac{y_{j, k+1}(t)-2 y_{j, k}(t)+y_{j, k-1}(t)}{h_{2}^{2}}+a_{j, k} y_{j, k}^{\prime}(t) \\
& -h_{1}^{2}\left(\frac{y_{j+1, k}^{\prime}(t)-2 y_{j, k}^{\prime}(t)+y_{j-1, k}^{\prime}(t)}{h_{1}^{2}}\right)-h_{2}^{2}\left(\frac{y_{j, k+1}^{\prime}(t)-2 y_{j, k}^{\prime}(t)+y_{j, k-1}^{\prime}(t)}{h_{2}^{2}}\right)=0 .
\end{aligned}
$$

In this work, we treat an important case left open in [24]. It is the one in which the model under consideration is posed in a regular 2-D bounded domain. This introduces important additional technical difficulties related with the analysis near the boundary. In this respect, we introduce a sequence of discretized domains $\left(\boldsymbol{\Omega}_{\boldsymbol{h}_{1}, \boldsymbol{h}_{\mathbf{2}}}\right)_{h_{1}, h_{2}}$ converging to $\Omega$ (in a sense that will be made precise later) when the mesh parameters $h_{1}$ and $h_{2}$ go to zero (see Fig. 1). Then, we associate to the first equation of (1) the finite-difference semi-discretization equation (11)

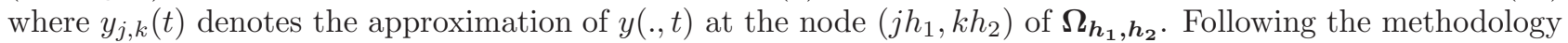
developed in [24], we show the efficiency of this scheme : we first prove that the energy associated with this numerical scheme decays exponentially, with a rate independent of the mesh size (see Th. 2.1, Sect. 2). To obtain the result we combine discrete multiplier methods introduced in [11] and compactness-uniqueness arguments. Let us note that a discrete version of multipliers was also developed in $[11,26]$ to address the issue of boundary observability in 1-D and the 2-D square domain. Then, we prove that the solution of the discretized system defined on $\boldsymbol{\Omega}_{\boldsymbol{h}_{1}, \boldsymbol{h}_{\mathbf{2}}}$ converges to the solution of (1) defined on $\Omega$ (see Th. 3.1, Sect. 3). Finally, we present some numerical experiments in order to confirm these theoretical results (Sect. 4).

\section{Discretization of the DOMAin $\Omega$ AND The Finite DifFEREnT SCHEME}

\subsection{Discretization of the domain $\Omega$}

We introduce in this paragraph a spatial discretization of the domain $\Omega$. Without loss of generality, we assume that the domain $\Omega$ is included in the unit square box: $\Omega \subseteq B \equiv] 0,1[\times] 0,1[$. For non negatives integer $J$ 

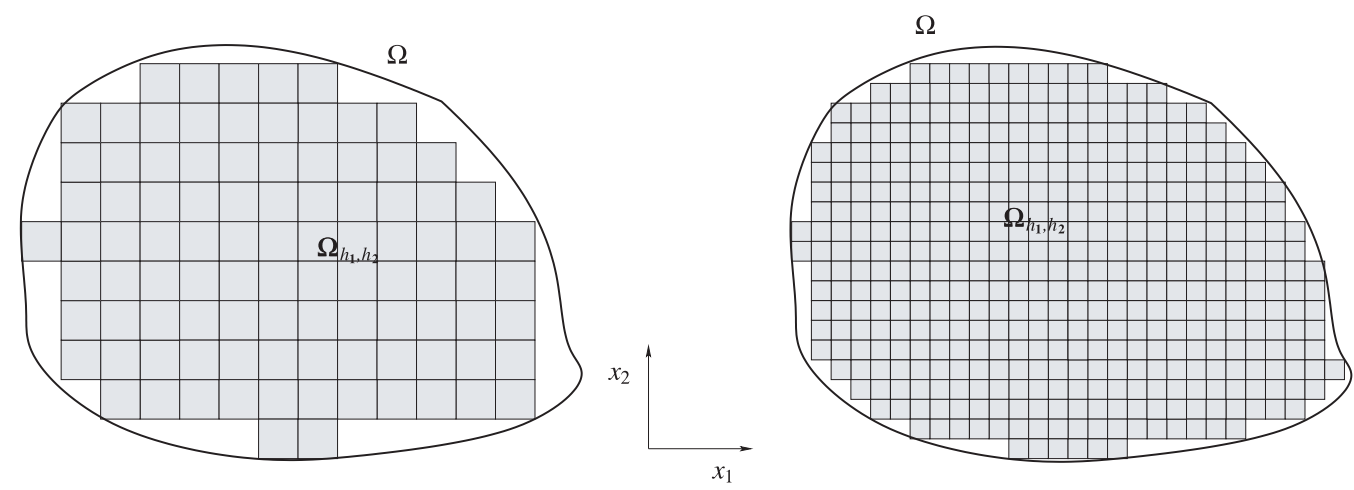

Figure 1. General domain $\Omega$ and two different discretizations $\boldsymbol{\Omega}_{\boldsymbol{h}_{1}, \boldsymbol{h}_{\mathbf{2}}}$.

and $K$, we then set

$$
h_{1}=\frac{1}{J+1}, \quad h_{2}=\frac{1}{K+1},
$$

and consider the two following uniform subdivisions of the interval $[0,1]$

$$
\left\{\begin{array}{l}
0=x_{1,0}<x_{1,1}<\ldots<x_{1, J}<x_{1, J+1}=1 \\
0=x_{2,0}<x_{2,1}<\ldots<x_{2, K}<x_{2, K+1}=1
\end{array}\right.
$$

where $x_{1, j}=j h_{1}$ and $x_{2, k}=k h_{2}$ for $j \in[0, J+1]$ and $k \in[0, K+1]$. We associate to the grid $G=\left(x_{1, j}, x_{2, k}\right)_{j, k}$ a discretization $\boldsymbol{B}_{\boldsymbol{h}_{1}, \boldsymbol{h}_{2}}$ of $B$ as follow:

$$
\boldsymbol{B}_{\boldsymbol{h}_{1}, \boldsymbol{h}_{\mathbf{2}}}=\cup_{j \in[0, J], k \in[0, K]} B_{j, k},
$$

where

$$
\left.B_{j, k}=\right] x_{1, j}, x_{1, j+1}[\times] x_{2, k}, x_{2, k+1}[
$$

represents the cell of area $h_{1} h_{2}$. Naturally, in the simple case of the unit square, the discretization is exact in the sense that $B_{h_{1}, h_{2}}=B$. We then define $\boldsymbol{\Omega}_{\boldsymbol{h}_{1}, \boldsymbol{h}_{\mathbf{2}}}$ as the union of all the cells $B_{j, k}$ strictly included in $\Omega$ :

$$
\boldsymbol{\Omega}_{\boldsymbol{h}_{\mathbf{1}}, \boldsymbol{h}_{\mathbf{2}}}=\cup_{j, k} B_{j, k}, \quad \forall j \in[0, J], k \in[0, K] \text { such that } B_{j, k} \subset \Omega
$$

implying in particular the inclusion $\boldsymbol{\Omega}_{\boldsymbol{h}_{1}, \boldsymbol{h}_{\mathbf{2}}} \subset \Omega$ for all $h_{1}$ and $h_{2}$. More precisely, we can write $\boldsymbol{\Omega}_{\boldsymbol{h}_{1}, \boldsymbol{h}_{\mathbf{2}}}$ in the following way

$$
\boldsymbol{\Omega}_{\boldsymbol{h}_{\mathbf{1}}, \boldsymbol{h}_{\mathbf{2}}}=\cup_{j=0, J} \cup_{k \in \mathcal{I}_{j}} B_{j, k}
$$

where, for $j$ from 0 to $J, \mathcal{I}_{j}$ designs the subset of $[0, K]$ defined by

$$
\mathcal{I}_{j}=\left\{k \in[0, K]: B_{j, k} \subset \boldsymbol{\Omega}_{\boldsymbol{h}_{1}, \boldsymbol{h}_{\mathbf{2}}}\right\}, \quad j \in[0, J] .
$$

Therefore, $k \in \mathcal{I}_{j} \Longleftrightarrow B_{j, k} \in \boldsymbol{\Omega}_{\boldsymbol{h}_{\mathbf{1}}, \boldsymbol{h}_{\mathbf{2}}}$. Now, in order to state the next definition, we extend $\mathcal{I}_{j}$ to $j=-1$ and $j=J+1$ putting $\mathcal{I}_{-1}=\emptyset$ and $\mathcal{I}_{J+1}=\emptyset$. Then, proceeding as before, we define the approximation $\boldsymbol{\omega}_{\boldsymbol{h}_{1}, \boldsymbol{h}_{\mathbf{2}}}$ of the open subset $\omega$ as the union of all the cells $B_{j, k}$ included in $\omega$.

Let us now describe the boundary of $\boldsymbol{\Omega}_{\boldsymbol{h}_{1}, \boldsymbol{h}_{\mathbf{2}}}$ which plays a crucial role in the context of stabilization. According to (16), the boundary is composed of segments $\tilde{I}(j, k)$ and $\hat{I}(j, k)$ parallel to the axis $O x_{1}$ or to $O x_{2}$, respectively:

$$
\widetilde{I}(j, k)=\{j\} \times[k, k+1] \text { and } \widehat{I}(j, k)=[j, j+1] \times\{k\} \text {, }
$$



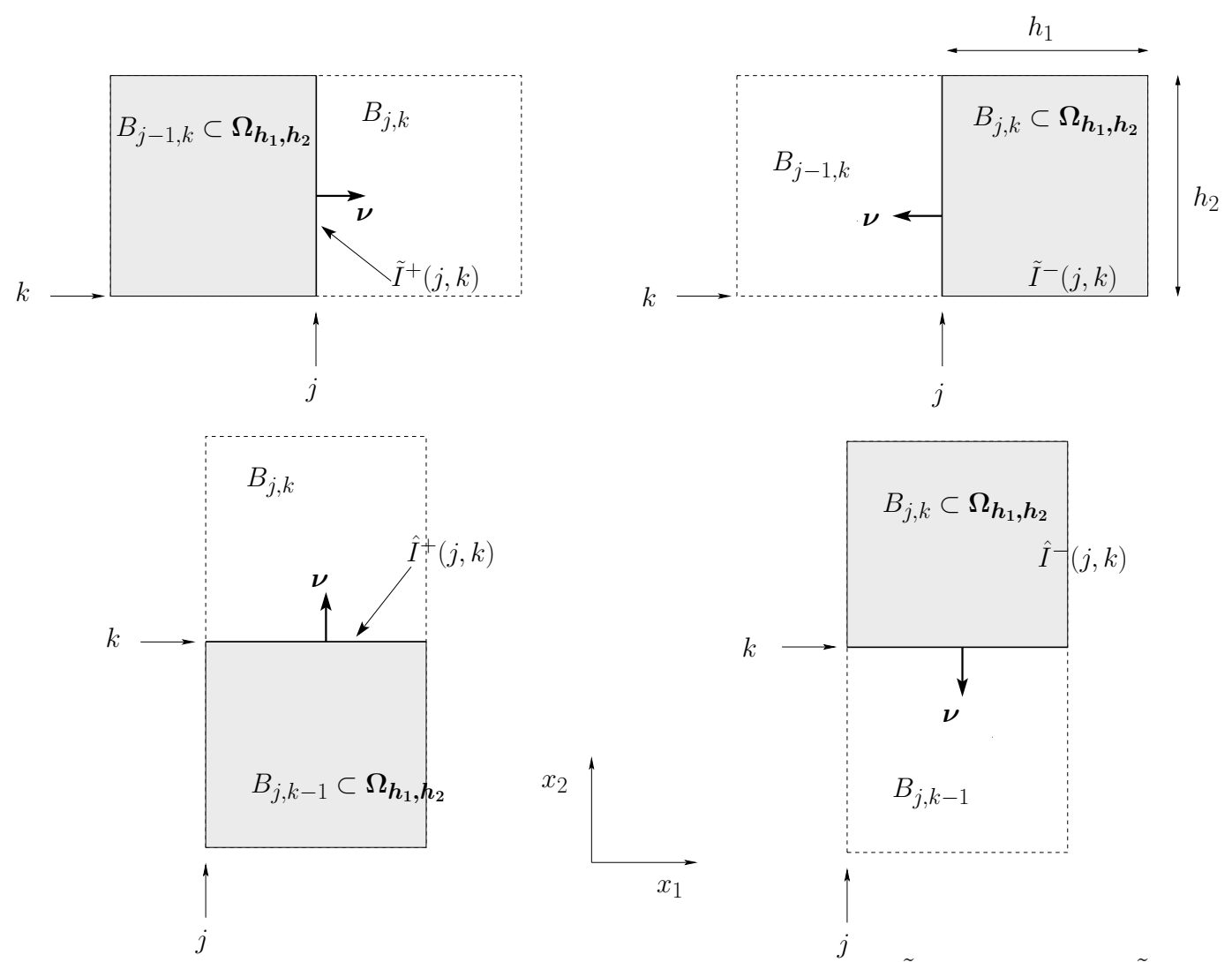

Figure 2. Description of the four types of segments of the boundary: $\tilde{I}^{+}(j, k)$ (left up), $\tilde{I}^{-}(j, k)$ (right up), $\hat{I}^{+}(j, k)$ (left down) and $\hat{I}^{-}(j, k)$ (right down).

for $j \in[0, J]$ and $k \in[0, K]$. Thus,

$$
\widetilde{I}(j, k) \subset \partial \boldsymbol{\Omega}_{\boldsymbol{h}_{1}, \boldsymbol{h}_{2}} \quad \text { if } \quad\left(k \in \mathcal{I}_{j} \text { and } k \notin \mathcal{I}_{j-1}\right) \quad \text { or } \quad\left(k \notin \mathcal{I}_{j} \text { and } k \in \mathcal{I}_{j-1}\right)
$$

and

$$
\widehat{I}(j, k) \subset \partial \boldsymbol{\Omega}_{\boldsymbol{h}_{1}, \boldsymbol{h}_{\mathbf{2}}} \quad \text { if } \quad\left(k \in \mathcal{I}_{j} \text { and } k-1 \notin \mathcal{I}_{j}\right) \quad \text { or } \quad\left(k \notin \mathcal{I}_{j} \text { and } k-1 \in \mathcal{I}_{j}\right) .
$$

In the sequel, we will need a finer distinction of the elements of the boundary of $\Omega_{h_{1}, h_{2}}$ :

Definition 1.1 (Boundary of $\boldsymbol{\Omega}_{\boldsymbol{h}_{1}, \boldsymbol{h}_{2}}$ ). We introduce the following subsets of $\mathbb{N} \times \mathbb{N}$ :

- $\tilde{I}^{+}=\left\{(j, k)\right.$ such that $\left.k \in \mathcal{I}_{j-1}, k \notin \mathcal{I}_{j}, j \in[1, J+1]\right\}$;

- $\tilde{I}^{-}=\left\{(j, k)\right.$ such that $\left.k \in \mathcal{I}_{j}, k \notin \mathcal{I}_{j-1}, j \in[0, J]\right\}$;

- $\hat{I}^{+}=\left\{(j, k)\right.$ such that $\left.k-1 \in \mathcal{I}_{j}, k \notin \mathcal{I}_{j}, j \in[0, J]\right\}$;

- $\hat{I}^{-}=\left\{(j, k)\right.$ such that $\left.k-1 \notin \mathcal{I}_{j}, k \in \mathcal{I}_{j}, j \in[0, J]\right\}$

describing the nodes of $\partial \boldsymbol{\Omega}_{\boldsymbol{h}_{1}, \boldsymbol{h}_{2}}$.

One element of each of these four subsets is described in Figure 2.

Remark 1.2. In the sequel, in order to simplify the notation, we will identify each of these four subsets by the corresponding union of segments of the boundary: for instance, we identify $\tilde{I}^{+}$with $\left\{\boldsymbol{x} \in \tilde{I}(j, k), \forall(j, k) \in \tilde{I}^{+}\right\}$.

To illustrate the parametrization above we write it explicitly for the simple polygonal domain $\boldsymbol{\Omega}_{\boldsymbol{h}_{1}, \boldsymbol{h}_{2}}$ described in the Figure 3 where $J=8$ and $K=5$. The objects corresponding to this example are summarized on 


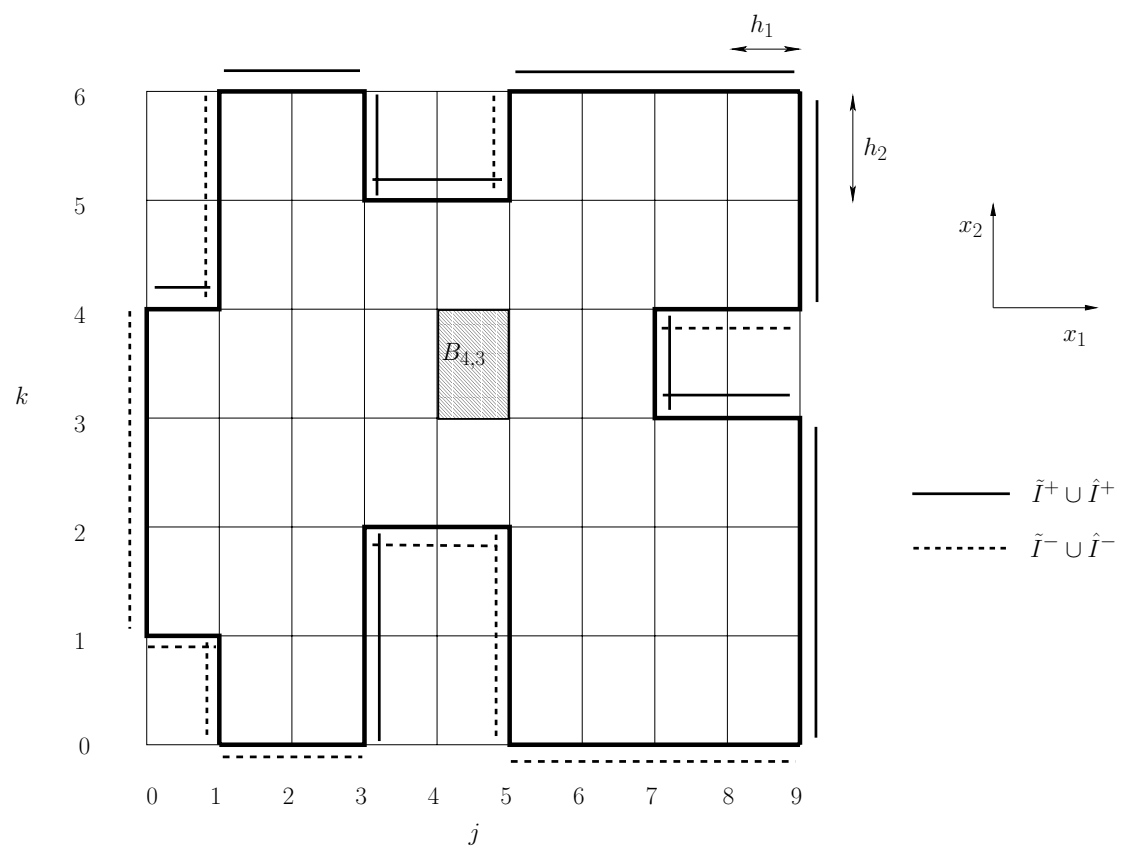

Figure 3. Polygonal domain $\boldsymbol{\Omega}_{\boldsymbol{h}_{\mathbf{1}}, \boldsymbol{h}_{\mathbf{2}}}$ with $J=8$ and $K=5$.

TABLE 1. Indexes corresponding to the parametrization of $\boldsymbol{\Omega}_{\boldsymbol{h}_{1}, \boldsymbol{h}_{\mathbf{2}}}$ in Figure 3.

\begin{tabular}{c|cc}
$j$ & $n(j)$ & $\mathcal{I}_{j}$ \\
\hline \hline 0 & 1 & $\{1,2,3\}$ \\
1 & 1 & $\{0,1,2,3,4,5\}$ \\
2 & 1 & $\{0,1,2,3,4,5\}$ \\
3 & 1 & $\{2,3,4\}$ \\
4 & 1 & $\{2,3,4\}$ \\
5 & 1 & $\{0,1,2,3,4,5\}$ \\
6 & 1 & $\{0,1,2,3,4,5\}$ \\
7 & 2 & $\{0,1,2\} \cup\{4,5\}$ \\
8 & 2 & $\{0,1,2\} \cup\{4,5\}$
\end{tabular}

Table 1 for which the subsets $\widetilde{I}^{+}$and $\widehat{I}^{+}$are

$$
\left\{\begin{array}{l}
\widetilde{I}^{+}=\{(3,0),(3,1),(3,5),(7,3),(9,0),(9,1),(9,2),(9,4),(9,5)\} \\
\widehat{I}^{+}=\{(0,4),(3,5),(4,5),(7,3),(8,3),(9,3),(1,6),(2,6),(5,6),(6,6),(7,6),(8,6)\} .
\end{array}\right.
$$

\subsection{Finite difference scheme}

Thanks to the parametrization described above, we are now in the position to introduce and study a discretized version of the system (1). We consider the following semi-discrete finite difference scheme resulting 
from the usual 5-points approximation (of order 2) in space of the laplacian:

$$
\left\{\begin{array}{r}
y_{j, k}^{\prime \prime}-\frac{y_{j+1, k}-2 y_{j, k}+y_{j-1, k}}{h_{1}^{2}}-\frac{y_{j, k+1}-2 y_{j, k}+y_{j, k-1}}{h_{2}^{2}}+a_{j, k} y_{j, k}^{\prime} \\
-h_{1}^{2}\left(\frac{y_{j+1, k}^{\prime}-2 y_{j, k}^{\prime}+y_{j-1, k}^{\prime}}{h_{1}^{2}}\right)-h_{2}^{2}\left(\frac{y_{j, k+1}^{\prime}-2 y_{j, k}^{\prime}+y_{j, k-1}^{\prime}}{h_{2}^{2}}\right)=0 \\
\quad \text { for }(j, k) \in \boldsymbol{\Omega}_{\boldsymbol{h}_{\mathbf{1}}, \boldsymbol{h}_{\mathbf{2}}} \text { and } t \in(0, \infty), \\
y_{j, k}(t)=0 \quad \text { for }(j, k) \in \partial \boldsymbol{\Omega}_{\boldsymbol{h}_{1}, \boldsymbol{h}_{\mathbf{2}}} \text { and } t \in(0, \infty), \\
y_{j, k}(t)=\left(y_{0}\right)_{j, k}, \quad y_{j, k}^{\prime}(t)=\left(y_{1}\right)_{j, k} \quad \text { for }(j, k) \in \boldsymbol{\Omega}_{\boldsymbol{h}_{\mathbf{1}}, \boldsymbol{h}_{\mathbf{2}}} \text { and } t=0 .
\end{array}\right.
$$

which differs from the most classical finite-difference scheme by the addition of viscous terms. We shall see below that this numerical approximation scheme converges in the energy space to the continuous damped wave equation (1).

Here, $y_{j, k}$ stands for the approximation of the solution $y$ at the mesh point $\left(x_{1, j}, x_{2, k}\right)$ and at time $t \in(0, \infty)$ and $\left(y_{0}\right)_{j, k}$ and $\left(y_{1}\right)_{j, k}$ the approximations of the initial data $y_{0}(\boldsymbol{x})$ and $y_{1}(\boldsymbol{x})$ at time $t=0$. Furthermore, the notation for $(j, k) \in \boldsymbol{\Omega}_{\boldsymbol{h}_{1}, \boldsymbol{h}_{2}}$ means for $j \in[0, J], k \in \mathcal{I}_{j}$, i.e. for the indexes $(j, k)$ such that the nodes $\left(x_{1, j}, x_{2, k}\right)$ are in $\boldsymbol{\Omega}_{\boldsymbol{h}_{\mathbf{1}}, \boldsymbol{h}_{\mathbf{2}}}$. In the same manner, the notation for $(j, k) \in \partial \boldsymbol{\Omega}_{\boldsymbol{h}_{\mathbf{1}}, \boldsymbol{h}_{\mathbf{2}}}$ means for $(j, k) \in \tilde{I}^{+} \cup \tilde{I}^{-} \cup$ $\hat{I}^{+} \cup \hat{I}^{-} . a_{j, k}$ denotes the approximation of the damping at the mesh point $\left(x_{1, j}, x_{2, k}\right)$ and we assume that there exists a positive constant $\alpha_{0}$ such that

$$
a_{j, k} \geq \alpha_{0} \forall(j, k) \in \boldsymbol{\omega}_{\boldsymbol{h}_{1}, \boldsymbol{h}_{2}} .
$$

According to $(7), \boldsymbol{\omega}_{\boldsymbol{h}_{\mathbf{1}}, \boldsymbol{h}_{\mathbf{2}}}$ defined in Section 1 is as follow

$$
\boldsymbol{\omega}_{\boldsymbol{h}_{1}, h_{2}} \text { is the intersection of } \boldsymbol{\Omega}_{\boldsymbol{h}_{1}, h_{2}} \text { and a neighborhood of } \tilde{I}^{+} \cup \hat{I}^{+} \text {. }
$$

We can associate to this scheme the following energy:

$$
E_{h_{1}, h_{2}}(t)=\frac{h_{1} h_{2}}{2} \sum_{j=0}^{J} \sum_{k \in \mathcal{I}_{j}}\left\{\left(y_{j, k}^{\prime}\right)^{2}+\left(\frac{y_{j+1, k}-y_{j, k}}{h_{1}}\right)^{2}+\left(\frac{y_{j, k+1}-y_{j, k}}{h_{2}}\right)^{2}\right\},
$$

which appears to be the semi-discrete version of the energy $E$ in (4) of the solutions of the continuous wave equation. Moreover, multiplying the first equation in (21) by $y_{j, k}^{\prime}$ and summing over $j$ and $k$, one can show that the discretized energy $E_{h_{1}, h_{2}}$ is a non increasing function of time. More precisely,

$$
\begin{aligned}
E_{h_{1}, h_{2}}^{\prime}(t)= & -h_{1} h_{2} \sum_{j=0}^{J} \sum_{k \in \mathcal{I}_{j}} a_{j, k}\left(y_{j, k}^{\prime}\right)^{2} \\
& -h_{1} h_{2} \sum_{j=0}^{J} \sum_{k \in \mathcal{I}_{j}}\left\{h_{1}^{2}\left(\frac{y_{j+1, k}^{\prime}-y_{j, k}^{\prime}}{h_{1}}\right)^{2}+h_{2}^{2}\left(\frac{y_{j, k+1}^{\prime}-y_{j, k}^{\prime}}{h_{2}}\right)^{2}\right\}
\end{aligned}
$$

which is a semi-discrete version of the energy dissipation law (5).

Furthermore, in order to obtain the exponential decay of solutions of the damped semi-discrete system (21) it is necessary and sufficient to prove the existence of a time $T>0$ and a constant $C>0$ independent of $h_{1}$ and $h_{2}$ such that the following Observability Inequality holds

$$
E_{h_{1}, h_{2}}(0) \leq C h_{1} h_{2} \sum_{j=0}^{J} \sum_{k \in \mathcal{I}_{j}} \int_{0}^{T} a_{j, k}\left(y_{j, k}^{\prime}\right)^{2} \mathrm{~d} t
$$

for every solution of (21). In the next section, we will proved this inequality using a discrete multiplier method. 
We highlight the fact that the constant $C$ in (26) may be not independent of $h_{1}, h_{2}$ for some solutions of the usual scheme without extra viscous terms. More precisely, the usual scheme creates high frequency spurious solutions that propagate very slowly (with group velocity of the order of the step size $h_{1}$ and $h_{2}$ ) making the observability property (26) impossible to hold uniformly in $h_{1}$ and $h_{2}$. The purpose of the viscous terms is to precisely absorb these high frequency solutions for which the quantities $y_{j+1, k}^{\prime}-2 y_{j, k}^{\prime}+y_{j-1, k}^{\prime}$ and $y_{j, k+1}^{\prime}-2 y_{j, k}^{\prime}+y_{j, k-1}^{\prime}$ are not negligible.

Remark 1.3. The first equation in (21) can be seen as a semi-discrete version of the following viscous wave equation defined on $\Omega$ :

$$
y^{\prime \prime}-\Delta y+a(\boldsymbol{x}) y^{\prime}-h_{1}^{2} \frac{\partial^{2}}{\partial x_{1}^{2}} y^{\prime}-h_{2}^{2} \frac{\partial^{2}}{\partial x_{2}^{2}} y^{\prime}=0 .
$$

Therefore, the extra viscous terms introduced are closely connected to the Tychonoff regularization technique [7] and also to the techniques introduced in $[13,17]$ which consist to add extra corrected terms proportional to $h_{1}^{2}, h_{2}^{2}$ in order to restore uniform observability property (see [27] for more details). Furthermore, a similar uniform result is obtained in [21] in the framework of the finite element approximation. In addition, it is proved that the power of $\boldsymbol{h}=\left(h_{1}, h_{2}\right)$ which appears in the extra viscous term is equal to the degree of approximation (in space) of the finite element method.

In the following, we denote by $C$ a positive constant that may vary from line to line but is independent on $h_{1}$ and $h_{2}$.

\section{UNIFORM STABILIZATION OF THE ENERGY}

This section is devoted to show that the energy associated to (21) decays exponentially, the rate being uniform with respect to the mesh size. Our main result reads as follows:

Theorem 2.1 (uniform exponential decay). Let $\left(a_{j, k}\right)$ and $\boldsymbol{\omega}_{\boldsymbol{h}_{1}, \boldsymbol{h}_{\mathbf{2}}}$ be given as in (22) and (23), respectively. Then, there exist two positive constants $C$ and $\alpha$ independent of $\left(h_{1}, h_{2}\right)$ such that the energy of the system $(21)$ satisfies

for every solution of (21).

$$
E_{h_{1}, h_{2}}(t) \leq C E_{h_{1}, h_{2}}(0) \mathrm{e}^{-\alpha t}, \forall t \geq 0, \forall h_{1}, h_{2} \in(0,1)
$$

Theorem 2.1 shows that the numerical viscosity term added in system (21) is enough to restore the uniform (with respect to $\left(h_{1}, h_{2}\right) \rightarrow 0$ ) exponential decay. This was already proved in [24] in $1-\mathrm{D}$ and $2-\mathrm{D}$ in the particular case of a square domain. Lately, this approach was used in [21] to construct uniformly exponentially stable approximations for an abstract class of second order evolution equations with bounded feedback controls.

The proof of Theorem 2.1 is divided in six steps: in Step 1, we first reduce the problem to obtaining a suitable uniform Observability Inequality for the undamped system (see (30) below). In Step 2, we establish a discrete boundary observability estimate (see (41)) leading in Step 3 to the discrete boundary observability (46). Step 4 is devoted to the absorption of the boundary terms leading in Step 5, to an internal observability inequality (56). Finally, in Step 6, using a compactness-uniqueness argument, we obtain the desired inequality (28).

\section{Proof of Theorem 2.1}

\section{Step 1. Reduction of the problem}

To prove the exponential decay of solutions of the damped semi-discrete system (21) it is necessary and sufficient to prove the existence of a time $T>0$ and a constant $C>0$ independent of $h_{1}$ and $h_{2}$ such that the following observability inequality holds

$$
E_{h_{1}, h_{2}}(0) \leq C h_{1} h_{2} \sum_{j=0}^{J} \sum_{k \in \mathcal{I}_{j}} \int_{0}^{T}\left[a_{j, k}\left(u_{j, k}^{\prime}\right)^{2}+h_{1}^{2}\left(\frac{u_{j+1, k}^{\prime}-u_{j, k}^{\prime}}{h_{1}}\right)^{2}+h_{2}^{2}\left(\frac{u_{j, k+1}^{\prime}-u_{j, k}^{\prime}}{h_{2}}\right)^{2}\right] \mathrm{d} t
$$


for every solution $\boldsymbol{u}_{\boldsymbol{h}_{1}, \boldsymbol{h}_{2}}$ of the conservative system

$$
\left\{\begin{array}{l}
u_{j, k}^{\prime \prime}-\frac{u_{j+1, k}-2 u_{j, k}+u_{j-1, k}}{h_{1}^{2}}-\frac{u_{j, k+1}-2 u_{j, k}+u_{j, k-1}}{h_{2}^{2}}=0 \\
\quad \text { for }(j, k) \in \boldsymbol{\Omega}_{\boldsymbol{h}_{\mathbf{1}}, \boldsymbol{h}_{\mathbf{2}}} \text { and } t \in(0, \infty), \\
u_{j, k}(t)=0 \text { for }(j, k) \in \partial \boldsymbol{\Omega}_{\boldsymbol{h}_{1}, \boldsymbol{h}_{\mathbf{2}}} \text { and } t \in(0, \infty), \\
u_{j, k}(0)=\left(y_{0}\right)_{j, k}, \quad u_{j, k}^{\prime}(0)=\left(y_{1}\right)_{j, k} \quad \text { for }(j, k) \in \boldsymbol{\Omega}_{\boldsymbol{h}_{\mathbf{1}}, \boldsymbol{h}_{\mathbf{2}}}
\end{array}\right.
$$

and all $0<h_{1}, h_{2}<1$.

Indeed, we argue as in [24]. Let us assume that (29) holds. We decompose the solution $\boldsymbol{y}_{\boldsymbol{h}_{\mathbf{1}}, \boldsymbol{h}_{\mathbf{2}}}$ of $(21)$ as $\boldsymbol{y}_{\boldsymbol{h}_{1}, \boldsymbol{h}_{2}}=\boldsymbol{u}_{\boldsymbol{h}_{1}, \boldsymbol{h}_{2}}+\boldsymbol{z}_{\boldsymbol{h}_{1}, \boldsymbol{h}_{2}}$ where $\boldsymbol{u}_{\boldsymbol{h}_{1}, \boldsymbol{h}_{2}}$ solves (30) and $\boldsymbol{z}_{\boldsymbol{h}_{1}, \boldsymbol{h}_{2}}$ is the solution of

$$
\left\{\begin{array}{c}
z_{j, k}^{\prime \prime}-\frac{z_{j+1, k}-2 z_{j, k}+z_{j-1, k}}{h_{1}^{2}}-\frac{z_{j, k+1}-2 z_{j, k}+z_{j, k-1}}{h_{2}^{2}} \\
=\left(y_{j+1, k}^{\prime}-2 y_{j, k}^{\prime}+y_{j-1, k}^{\prime}\right)+\left(y_{j, k+1}^{\prime}-2 y_{j, k}^{\prime}+y_{j, k-1}^{\prime}\right)-a_{j, k} y_{j, k}^{\prime} \\
\text { for }(j, k) \in \boldsymbol{\Omega}_{\boldsymbol{h}_{\mathbf{1}}, \boldsymbol{h}_{\mathbf{2}}} \text { and } t \in(0, \infty), \\
z_{j, k}(t)=0 \quad \text { for }(j, k) \in \partial \boldsymbol{\Omega}_{\boldsymbol{h}_{\mathbf{1}}, \boldsymbol{h}_{\mathbf{2}}} \text { and } t \in(0, \infty), \\
z_{j, k}(0)=0, \quad z_{j, k}^{\prime}(0)=0 \text { for }(j, k) \in \boldsymbol{\Omega}_{\boldsymbol{h}_{\mathbf{1}}, \boldsymbol{h}_{\mathbf{2}} .}
\end{array}\right.
$$

According to (29) we have

$$
\begin{aligned}
E_{h_{1}, h_{2}}(0) \leq & C h_{1} h_{2} \sum_{j=0}^{J} \sum_{k \in \mathcal{I}_{j}} \int_{0}^{T}\left[a_{j, k}\left(y_{j, k}^{\prime}\right)^{2}+h_{1}^{2}\left(\frac{y_{j+1, k}^{\prime}-y_{j, k}^{\prime}}{h_{1}}\right)^{2}+h_{2}^{2}\left(\frac{y_{j, k+1}^{\prime}-y_{j, k}^{\prime}}{h_{2}}\right)^{2}\right. \\
& \left.+a_{j, k}\left(z_{j, k}^{\prime}\right)^{2}+h_{1}^{2}\left(\frac{z_{j+1, k}^{\prime}-z_{j, k}^{\prime}}{h_{1}}\right)^{2}+h_{2}^{2}\left(\frac{z_{j, k+1}^{\prime}-z_{j, k}^{\prime}}{h_{2}}\right)^{2}\right] \mathrm{d} t .
\end{aligned}
$$

Let us now assume for the moment that the following inequality holds:

$$
\begin{aligned}
\sum_{j=0}^{J} \sum_{k \in \mathcal{I}_{j}} & \int_{0}^{T}\left[a_{j, k}\left(z_{j, k}^{\prime}\right)^{2}+h_{1}^{2}\left(\frac{z_{j+1, k}^{\prime}-z_{j, k}^{\prime}}{h_{1}}\right)^{2}+h_{2}^{2}\left(\frac{z_{j, k+1}^{\prime}-z_{j, k}^{\prime}}{h_{2}}\right)^{2}\right] \mathrm{d} t \\
& \leq C \sum_{j=0}^{J} \sum_{k \in \mathcal{I}_{j}} \int_{0}^{T}\left[a_{j, k}\left(y_{j, k}^{\prime}\right)^{2}+h_{1}^{2}\left(\frac{y_{j+1, k}^{\prime}-y_{j, k}^{\prime}}{h_{1}}\right)^{2}+h_{2}^{2}\left(\frac{y_{j, k+1}^{\prime}-y_{j, k}^{\prime}}{h_{2}}\right)^{2}\right] \mathrm{d} t .
\end{aligned}
$$

Combining (32) and (33), it follows that

$$
\begin{aligned}
E_{h_{1}, h_{2}}(T) & \leq E_{h_{1}, h_{2}}(0) \\
& \leq C h_{1} h_{2} \sum_{j=0} \sum_{k \in \mathcal{I}_{j}} \int_{0}^{T}\left[a_{j, k}\left(y_{j, k}^{\prime}\right)^{2}+h_{1}^{2}\left(\frac{y_{j+1, k}^{\prime}-y_{j, k}^{\prime}}{h_{1}}\right)^{2}+h_{2}^{2}\left(\frac{y_{j, k+1}^{\prime}-y_{j, k}^{\prime}}{h_{2}}\right)^{2}\right] \mathrm{d} t .
\end{aligned}
$$

Now, integrating in $(0, T)$ the energy dissipation law (25) for solutions of (21), it follows that

$$
E_{h_{1}, h_{2}}(T)-E_{h_{1}, h_{2}}(0)=-h_{1} h_{2} \sum_{j=0}^{J} \sum_{k \in \mathcal{I}_{j}} \int_{0}^{T}\left[a_{j, k}\left(y_{j, k}^{\prime}\right)^{2}+h_{1}^{2}\left(\frac{y_{j+1, k}^{\prime}-y_{j, k}^{\prime}}{h_{1}}\right)^{2}+h_{2}^{2}\left(\frac{y_{j, k+1}^{\prime}-y_{j, k}^{\prime}}{h_{2}}\right)^{2}\right] \mathrm{d} t .
$$

Combining (34) and (35), we deduce that

$$
E_{h_{1}, h_{2}}(T) \leq \gamma E_{h_{1}, h_{2}}(0)
$$


with $\gamma=\frac{C}{C+1}$. Inequality (36) together with the semigroup property imply that

$$
E_{h_{1}, h_{2}}(t) \leq \frac{1}{\gamma} \exp \frac{-|\log (\gamma)|}{T} t E_{h_{1}, h_{2}}(0), \forall t>0, \text { and } 0<h_{1}, h_{2}<1
$$

This yields the desired exponential decay.

To conclude, let us now check (33). Multiplying the equation satisfied by $\boldsymbol{z}_{\boldsymbol{h}_{1}, \boldsymbol{h}_{\mathbf{2}}}$ by $z_{j, k}^{\prime}$, we get

$$
\begin{aligned}
F_{h_{1}, h_{2}}^{\prime}(t)= & h_{1} h_{2} \sum_{j=0}^{J} \sum_{k \in \mathcal{I}_{j}}\left(h_{1}\left(\frac{y_{j+1, k}^{\prime}-y_{j, k}^{\prime}}{h_{1}}\right)+h_{2}\left(\frac{y_{j, k+1}^{\prime}-y_{j, k}^{\prime}}{h_{2}}\right)\right) z_{j, k}^{\prime} \\
& -h_{1} h_{2} \sum_{j=0}^{J} \sum_{k \in \mathcal{I}_{j}}\left(h_{1}\left(\frac{y_{j+1, k}^{\prime}-y_{j, k}^{\prime}}{h_{1}}\right) z_{j+1, k}^{\prime}+h_{2}\left(\frac{y_{j, k+1}^{\prime}-y_{j, k}^{\prime}}{h_{2}}\right) z_{j, k+1}^{\prime}\right) \\
& -h_{1} h_{2} \sum_{j=0}^{J} \sum_{k \in \mathcal{I}_{j}} a_{j, k} y_{j, k}^{\prime} z_{j, k}^{\prime} \\
\leq & F_{h_{1}, h_{2}}(t)+C h_{1} h_{2} \sum_{j=0}^{J} \sum_{k \in \mathcal{I}_{j}}\left(h_{1}^{2}\left(\frac{y_{j+1, k}^{\prime}-y_{j, k}^{\prime}}{h_{1}}\right)^{2}+h_{2}^{2}\left(\frac{y_{j, k+1}^{\prime}-y_{j, k}^{\prime}}{h_{2}}\right)^{2}+a_{j, k}\left(y_{j, k}^{\prime}\right)^{2}\right)
\end{aligned}
$$

where $F_{h_{1}, h_{2}}$ denotes the energy of the solution of system (31).

Integrating (38) in $[0, T]$, taking into account that $F_{h_{1}, h_{2}}(0)=0$, using the Gronwall lemma and the fact that

$$
\begin{gathered}
h_{1} h_{2} \sum_{j=0}^{J} \sum_{k \in \mathcal{I}_{j}} \int_{0}^{T}\left(h_{1}^{2}\left(\frac{z_{j+1, k}-z_{j, k}}{h_{1}}\right)^{2}+h_{2}^{2}\left(\frac{z_{j, k+1}-z_{j, k}}{h_{2}}\right)^{2}\right) \mathrm{d} t \\
\quad+h_{1} h_{2} \sum_{j=1}^{J} \sum_{k \in \mathcal{I}_{j}} \int_{0}^{T} a_{j, k}\left(z_{j, k}(t)\right)^{2} d t \leq C \int_{0}^{T} F_{h_{1}, h_{2}}(t) \mathrm{d} t
\end{gathered}
$$

inequality (33) immediately follows.

Remark 2.2. In the case where the damping is effective on the whole domain $(\omega=\Omega)$, we obtain easily that

$$
\sum_{j=0}^{J} \sum_{k \in \mathcal{I}_{j}} \int_{0}^{T}\left[h_{1}^{2}\left(\frac{u_{j+1, k}^{\prime}-u_{j, k}^{\prime}}{h_{1}}\right)^{2}+h_{2}^{2}\left(\frac{u_{j, k+1}^{\prime}-u_{j, k}^{\prime}}{h_{2}}\right)^{2}\right] \mathrm{d} t \leq C \sum_{j=0}^{J} \sum_{k \in \mathcal{I}_{j}} \int_{0}^{T} a_{j, k}\left(u_{j, k}^{\prime}\right)^{2} \mathrm{~d} t
$$

implying that the proof of (29) is reduced to the proof of (26). Therefore, in this case, the use of extra viscous terms is no longer necessary.

Step 2. Energy identity for the conservative discretized equation (30)

The purpose of this step is to derive an energy identity for the conservative discretized system (30), which is the basic tool for proving an a priori asymptotic estimate on the solution of (21). We state the result in the following lemma: 
Lemma 2.3. The semi-discretized energy $E_{h_{1}, h_{2}}$ satisfies the following relation:

$$
\begin{aligned}
& T E_{h_{1}, h_{2}}(0)=\int_{0}^{T} E_{h_{1}, h_{2}}(t) \mathrm{d} t \\
& \left.=-\frac{h_{1} h_{2}}{2} \sum_{j=0}^{J} \sum_{k \in \mathcal{I}_{j}} u_{j, k}^{\prime}\left(j h_{1}\left(\frac{u_{j+1, k}-u_{j-1, k}}{h_{1}}\right)+\frac{u_{j, k}}{2}\right)\right]_{0}^{T} \\
& \left.-\frac{h_{1} h_{2}}{2} \sum_{j=0}^{J} \sum_{k \in \mathcal{I}_{j}} u_{j, k}^{\prime}\left(k h_{2}\left(\frac{u_{j, k+1}-u_{j, k-1}}{h_{2}}\right)+\frac{u_{j, k}}{2}\right)\right]_{0}^{T} \\
& -\frac{h_{2}}{2} \sum_{(j, k) \in \tilde{I}^{-}} j h_{1} \int_{0}^{T}\left(\frac{u_{j+1, k}}{h_{1}}\right)^{2} \mathrm{~d} t+\frac{h_{2}}{2} \sum_{(j, k) \in \tilde{I}^{+}} j h_{1} \int_{0}^{T}\left(\frac{u_{j-1, k}}{h_{1}}\right)^{2} \mathrm{~d} t \\
& -\frac{h_{1}}{2} \sum_{(j, k) \in \hat{I}^{-}} k h_{2} \int_{0}^{T}\left(\frac{u_{j, k+1}}{h_{2}}\right)^{2} \mathrm{~d} t+\frac{h_{1}}{2} \sum_{(j, k) \in \hat{I}^{+}} k h_{2} \int_{0}^{T}\left(\frac{u_{j, k-1}}{h_{2}}\right)^{2} \mathrm{~d} t \\
& -\frac{h_{1} h_{2}}{2} \sum_{j=0}^{J} \sum_{k \in \mathcal{I}_{j}} \int_{0}^{T}\left\{\left(\frac{u_{j+1, k}-u_{j, k}}{h_{1}}\right)^{2}+\left(\frac{u_{j, k+1}-u_{j, k}}{h_{2}}\right)^{2}\right\} \mathrm{d} t \\
& +\frac{h_{1} h_{2}}{2} \sum_{j=0}^{J} \sum_{k \in \mathcal{I}_{j}} \int_{0}^{T}\left\{\left(\frac{u_{j+1, k+1}-u_{j, k+1}}{h_{1}}\right)\left(\frac{u_{j+1, k}-u_{j, k}}{h_{1}}\right)\right\} \mathrm{d} t \\
& +\frac{h_{1} h_{2}}{2} \sum_{j=0}^{J} \sum_{k \in \mathcal{I}_{j}} \int_{0}^{T}\left\{\left(\frac{u_{j+1, k+1}-u_{j+1, k}}{h_{2}}\right)\left(\frac{u_{j, k+1}-u_{j, k}}{h_{2}}\right)\right\} \mathrm{d} t \\
& +\frac{h_{1} h_{2}}{4} \sum_{j=0}^{J} \sum_{k \in \mathcal{I}_{j}} \int_{0}^{T}\left\{h_{1}^{2}\left(\frac{u_{j+1, k}^{\prime}-u_{j, k}^{\prime}}{h_{1}}\right)^{2}+h_{2}^{2}\left(\frac{u_{j, k+1}^{\prime}-u_{j, k}^{\prime}}{h_{2}}\right)^{2}\right\} \mathrm{d} t .
\end{aligned}
$$

Remark 2.4. The identity (41) is analogous - on a semi discrete level - to the well-known relation

$$
T E(0)=-\left.\int_{\Omega} u_{t}\left(\boldsymbol{x} \cdot \nabla u+\frac{u}{2}\right)\right|_{0} ^{T} \mathrm{~d} x+\frac{1}{2} \int_{0}^{T} \int_{\partial \Omega} \boldsymbol{x} \cdot \boldsymbol{\nu}\left|\frac{\partial u}{\partial \nu}\right|^{2} \mathrm{~d} \nu \mathrm{d} t
$$

where $u$ is solution the continuous conservative wave equation (1) with $a \equiv 0$. This relation may be obtained using the multipliers $\boldsymbol{x} \cdot \nabla u$ and $u$ (see [12] for instance). More precisely, the two first sums of (41) clearly approximate the first integral of (42). Furthermore, the third and fourth sums approximate the boundary terms of (42). To see this, it is convenient to split the boundary integral into two integrals in the subsets where $\boldsymbol{x} . \boldsymbol{\nu}>0$ and $\boldsymbol{x} . \boldsymbol{\nu}<0$, respectively. Due the Dirichlet boundary condition, the approximation of, for instance, $\frac{\partial u}{\partial \nu}$ on the point $\left(x_{1, j}, x_{2, k}\right)$ of $\tilde{I}^{+}$is simply $-\frac{u_{j-1, k}}{h_{1}}$. Furthermore, $\tilde{I}^{+} \cup \hat{I}^{+}$coincides with $\Gamma_{0}$ while $\tilde{I}^{-} \cup \hat{I}^{-}$ with $\partial \Omega \backslash \Gamma_{0}$. However, (41) contains some terms (the last four) that do not appear in the continuous case. It is precisely in order to absorb these terms and more specifically the positives ones that adding the numerical viscosity term is needed.

Proof of Lemma 2.3. Let us introduce $M_{j, k}$

$$
M_{j, k}=j\left(\frac{u_{j+1, k}-u_{j-1, k}}{2}\right)+k\left(\frac{u_{j, k+1}-u_{j, k-1}}{2}\right),
$$

which corresponds to the semi-discrete version of the multiplier $\boldsymbol{x}$. $\nabla u$. Following the developments in [24], we multiply the first equation of (30) by $M_{j, k}$, take the sum over $j$ and $k$, and integrate by parts in $[0, T]$. 
Step 3. Absorption of the term at time $t=0, T$ and the crossed one

Now we are going to estimate the term at time $t=0, T$ as well as the crossed terms entering in the right hand side of (41). Using Young inequality as well as (24), we obtain (see the appendix of [24] for more details concerning the unit square domain)

$$
\begin{aligned}
\left.\mid-\frac{h_{1} h_{2}}{2} \sum_{j=0}^{J} \sum_{k \in \mathcal{I}_{j}} u_{j, k}^{\prime}\left(j h_{1}\left(\frac{u_{j+1, k}-u_{j-1, k}}{h_{1}}\right)+\frac{u_{j, k}}{2}\right)\right]_{0}^{T} & \\
& \left.-\frac{h_{1} h_{2}}{2} \sum_{j=0}^{J} \sum_{k \in \mathcal{I}_{j}} u_{j, k}^{\prime}\left(k h_{2}\left(\frac{u_{j, k+1}-u_{j, k-1}}{h_{1}}\right)+\frac{u_{j, k}}{2}\right)\right]\left._{0}^{T}\right|^{2} \\
\leq & \leq\left(2 \sqrt{2}+\frac{\sqrt{2}}{4} \max \left(h_{1}^{2}, h_{2}^{2}\right)\right) E_{h_{1}, h_{2}}(0) .
\end{aligned}
$$

Also, we obtain

$$
\begin{aligned}
-\frac{h_{1} h_{2}}{2} \sum_{j=0}^{J} \sum_{k \in \mathcal{I}_{j}} \int_{0}^{T}\{( & \left.\left.\frac{u_{j+1, k}-u_{j, k}}{h_{1}^{2}}\right)^{2}+\left(\frac{u_{j, k+1}-u_{j, k}}{h_{2}^{2}}\right)^{2}\right\} \mathrm{d} t \\
& +\frac{h_{1} h_{2}}{2} \sum_{j=0}^{J} \sum_{k \in \mathcal{I}_{j}} \int_{0}^{T}\left\{\left(\frac{u_{j+1, k+1}-u_{j, k+1}}{h_{1}}\right)\left(\frac{u_{j+1, k}-u_{j, k}}{h_{1}}\right)\right\} \mathrm{d} t \\
& +\frac{h_{1} h_{2}}{2} \sum_{j=0}^{J} \sum_{k \in \mathcal{I}_{j}} \int_{0}^{T}\left\{\left(\frac{u_{j+1, k+1}-u_{j+1, k}}{h_{2}}\right)\left(\frac{u_{j, k+1}-u_{j, k}}{h_{2}}\right)\right\} \mathrm{d} t \leq 0
\end{aligned}
$$

The proof of this inequality relies essentially on the inequality $-\left(a^{2}+b^{2}\right)+2 a b \leq 0$, for all $a, b \in \mathbb{R}$. Reporting (44) and the above inequality in (41), we get for all $T>2 \sqrt{2}+\frac{\sqrt{2}}{4} \max \left(h_{1}^{2}, h_{2}^{2}\right)$

$$
\begin{aligned}
& \left(T-2 \sqrt{2}-\frac{\sqrt{2}}{4} \max \left(h_{1}^{2}, h_{2}^{2}\right)\right) E_{h_{1}, h_{2}}(0) \\
& \leq \frac{h_{1} h_{2}}{2} \sum_{(j, k) \in \tilde{I}^{+}} j \int_{0}^{T}\left(\frac{u_{j-1, k}}{h_{1}}\right)^{2} \mathrm{~d} t+\frac{h_{1} h_{2}}{2} \sum_{(j, k) \in \hat{I}^{+}} k \int_{0}^{T}\left(\frac{u_{j, k-1}}{h_{2}}\right)^{2} \mathrm{~d} t \\
& \quad+\frac{h_{1} h_{2}}{4} \sum_{j=0}^{J} \sum_{k \in \mathcal{I}_{j}} \int_{0}^{T}\left\{h_{1}^{2}\left(\frac{u_{j+1, k}^{\prime}-u_{j, k}^{\prime}}{h_{1}}\right)^{2}+h_{2}^{2}\left(\frac{u_{j, k+1}^{\prime}-u_{j, k}^{\prime}}{h_{2}}\right)^{2} \mathrm{~d} t\right.
\end{aligned}
$$

In (46) the boundary terms corresponding to the subsets $\tilde{I}^{-}$and $\hat{I}^{-}$have been neglected since they are negative. The next steps are devoted to the absorption of the remaining boundary terms in the right hand side of (46).

Step 4. Absorption of the boundary terms

Let us introduce the function $\boldsymbol{f}=\left(f^{1}, f^{2}\right) \in\left(W^{1, \infty}(\Omega)\right)^{2}$ of support $\hat{\omega}$ localized on the neighborhood of $\Gamma_{0}$ (defined in (8)) and included in $\omega$; more precisely, we write:

$$
\left\{\begin{array}{l}
f^{1}(\boldsymbol{x})=1 \text { on }\left\{\boldsymbol{x} \in \Gamma_{0}, \nu(\boldsymbol{x})=(1,0)\right\} \\
f^{2}(\boldsymbol{x})=1 \text { on }\left\{\boldsymbol{x} \in \Gamma_{0}, \nu(\boldsymbol{x})=(0,1)\right\} \\
\boldsymbol{f}(\boldsymbol{x})=\mathbf{0} \text { in } \Omega \backslash \mathcal{V}_{r}\left(\Gamma_{0}\right)
\end{array}\right.
$$


where $\mathcal{V}_{r}\left(\Gamma_{0}\right)$ denotes a neighborhood of $\Gamma_{0}$ for the euclidean distance,

$$
\mathcal{V}_{r}\left(\Gamma_{0}\right)=\left\{\boldsymbol{x} \in \Omega ; d\left(\boldsymbol{x}, \Gamma_{0}\right) \leq r\right\}
$$

and $r$ a sufficiently small positive real. We then define the discrete cut-off function of support the grid $G\left(\hat{\boldsymbol{\omega}}_{\boldsymbol{h}_{1}, \boldsymbol{h}_{\mathbf{2}}}\right)$ of $\hat{\boldsymbol{\omega}}_{\boldsymbol{h}_{\mathbf{1}}, \boldsymbol{h}_{\mathbf{2}}}$ defined as the union of cells $B_{j, k}$ contained in $\hat{\boldsymbol{\omega}}$ :

$$
\left\{\begin{array}{l}
f_{j, k}^{1}=1 \quad \text { if } \quad(j, k) \in \tilde{I}^{+}, \quad f_{j, k}^{2}=1 \quad \text { if } \quad(j, k) \in \hat{I}^{+}, \\
\boldsymbol{f}_{j, k}=0 \quad \text { if } \quad(j, k) \notin G\left(\boldsymbol{\Omega}_{\boldsymbol{h}_{\mathbf{1}}, \boldsymbol{h}_{\mathbf{2}}}\right) \backslash\left(\mathcal{V}_{r}\left(\tilde{I}^{+} \cup \hat{I}^{+}\right)\right) .
\end{array}\right.
$$

We also introduce a smooth nonnegative function $g=g(t)$ such that

$$
g(0)=g(T)=0, g(t)=1 \text { in }[\delta, T-\delta]
$$

where $\delta>0$ is sufficiently small. Now, we multiply the first equation in (30) by

$$
f_{j, k}^{1}\left(\frac{u_{j+1, k}-u_{j-1, k}}{2 h_{1}}\right) g(t)+f_{j, k}^{2}\left(\frac{u_{j, k+1}-u_{j, k-1}}{2 h_{2}}\right) g(t)
$$

and proceed as in the first step. We find

$$
\begin{aligned}
\frac{1}{2} \sum_{(j, k) \in \tilde{I}^{+}} & j \int_{0}^{T} g(t)\left(\frac{u_{j-1, k}}{h_{1}}\right)^{2} \mathrm{~d} t+\frac{1}{2} \sum_{(j, k) \in \hat{I}^{+}} k \int_{0}^{T} g(t)\left(\frac{u_{j, k-1}}{h_{2}}\right)^{2} \mathrm{~d} t \\
& =\frac{1}{2} \sum_{(j, k) \in \hat{\boldsymbol{\omega}}_{h_{1}, h_{2}}}\left(\frac{f_{j+1, k}^{1}-f_{j, k}^{1}}{h_{1}} \int_{0}^{T} g(t) u_{j+1, k}^{\prime} u_{j, k}^{\prime} \mathrm{d} t+\frac{f_{j, k+1}^{2}-f_{j, k}^{2}}{h_{2}} \int_{0}^{T} g(t) u_{j, k+1}^{\prime} u_{j, k}^{\prime} \mathrm{d} t\right) \\
& -\sum_{(j, k) \in \hat{\boldsymbol{\omega}}_{h_{1}, h_{2}}}\left(f_{j, k}^{1} \int_{0}^{T} g^{\prime}(t) u_{j, k}^{\prime}(t)\left(\frac{u_{j+1, k}-u_{j-1, k}}{2 h_{1}}\right) \mathrm{d} t+f_{j, k}^{2} \int_{0}^{T} g^{\prime}(t) u_{j, k}^{\prime}\left(\frac{u_{j, k+1}-u_{j, k-1}}{2 h_{2}}\right) \mathrm{d} t\right) \\
& +\frac{1}{2} \sum_{(j, k) \in \hat{\boldsymbol{\omega}}_{h_{1}, h_{2}}} \frac{f_{j+1, k}^{1}-f_{j, k}^{1}}{h_{1}} \int_{0}^{T} g(t)\left(\frac{u_{j+1, k}-u_{j, k}}{h_{1}}\right)^{2} \mathrm{~d} t \\
& +\frac{1}{2} \sum_{(j, k) \in \hat{\boldsymbol{\omega}}_{h_{1}, h_{2}}} \frac{f_{j, k+1}^{2}-f_{j, k}^{2}}{h_{2}} \int_{0}^{T} g(t)\left(\frac{u_{j, k+1}-u_{j, k}}{h_{2}}\right)^{2} \mathrm{~d} t \\
& -\sum_{(j, k) \in \hat{\boldsymbol{\omega}}_{h_{1}, h_{2}}} f_{j, k}^{2} \int_{0}^{T} g(t)\left(\frac{u_{j+1, k}-2 u_{j, k}+u_{j-1, k}}{h_{1}^{2}}\right)\left(\frac{u_{j, k+1}-u_{j, k-1}}{2 h_{2}}\right) \mathrm{d} t \\
& -\sum_{(j, k) \in \hat{\boldsymbol{\omega}}_{h_{1}, h_{2}}} f_{j, k}^{1} \int_{0}^{T} g(t)\left(\frac{u_{j, k+1}-2 u_{j, k}+u_{j, k-1}}{h_{2}^{2}}\right)\left(\frac{u_{j+1, k}-u_{j-1, k}}{2 h_{1}}\right) \mathrm{d} t .
\end{aligned}
$$

From the relation (52), we have the following result: 
Lemma 2.5. The bounded terms can be bounded as follows:

$$
\begin{aligned}
\frac{1}{2} \sum_{(j, k) \in \tilde{I}^{-}} j \int_{\delta}^{T-\delta}\left(\frac{u_{j-1, k}}{h_{1}}\right)^{2} \mathrm{~d} t & +\frac{1}{2} \sum_{(j, k) \in \hat{I}^{+}} k \int_{\delta}^{T-\delta}\left(\frac{u_{j, k-1}}{h_{2}}\right)^{2} \mathrm{~d} t \\
\leq C \sum_{j=0}^{J} \sum_{k \in \mathcal{I}_{j}} a_{j, k} \int_{0}^{T}\left(u_{j, k}^{\prime}(t)\right)^{2} \mathrm{~d} t+C \sum_{(j, k) \in \hat{\boldsymbol{\omega}}_{h_{1}, h_{2}}} \int_{0}^{T} g(t)\left(u_{j, k}(t)\right)^{2} \mathrm{~d} t & +C \sum_{(j, k) \in \hat{\boldsymbol{\omega}}_{h_{1}, h_{2}}} \int_{0}^{T} g(t)\left\{\left(\frac{u_{j+1, k}-u_{j, k}}{h_{1}}\right)^{2}+\left(\frac{u_{j, k+1}-u_{j, k}}{h_{2}}\right)^{2}\right\} \mathrm{d} t
\end{aligned}
$$

Proof of Lemma 2.5. Performing as in [24] the result follows.

Reporting (53) in (46) with $T>2 \sqrt{2}+\frac{\sqrt{2}}{4} \max \left(h_{1}^{2}, h_{2}^{2}\right)$ and $\delta$ small enough such that $T-2 \delta>0$, we obtain, using the invariance by the translation in time,

$$
\begin{aligned}
E_{h_{1}, h_{2}}(0) & \leq C h_{1} h_{2} \sum_{j=0}^{J} \sum_{k \in \mathcal{I}_{j}} \int_{0}^{T} a_{j, k}\left(u_{j, k}^{\prime}(t)\right)^{2} \mathrm{~d} t \\
& +C h_{1} h_{2} \sum_{j=0}^{J} \sum_{k \in \mathcal{I}_{j}} \int_{0}^{T}\left\{h_{1}^{2}\left(\frac{u_{j+1, k}^{\prime}-u_{j, k}^{\prime}}{h_{1}}\right)^{2}+h_{2}^{2}\left(\frac{u_{j, k+1}^{\prime}-u_{j, k}^{\prime}}{h_{2}}\right)^{2}\right\} \mathrm{d} t \\
& +C h_{1} h_{2} \sum_{(j, k) \in \hat{\boldsymbol{\omega}}_{h_{1}, h_{2}}} \int_{0}^{T} g(t)\left(u_{j, k}(t)\right)^{2} \mathrm{~d} t \\
& +C h_{1} h_{2} \sum_{(j, k) \in \hat{\boldsymbol{\omega}}_{h_{1}, h_{\mathbf{2}}}} \int_{0}^{T} g(t)\left\{\left(\frac{u_{j+1, k}-u_{j, k}}{h_{1}}\right)^{2}+\left(\frac{u_{j, k+1}-u_{j, k}}{h_{2}}\right)^{2}\right\} \mathrm{d} t .
\end{aligned}
$$

It remains to show that the last two terms in the right hand side of (54) may be uniformly absorbed:

Step 5. Absorption of the discrete gradient term

To absorb the last term in (54) which corresponds to the energy localized in the discrete gradient of $\hat{\boldsymbol{\omega}}_{\boldsymbol{h}_{1}, \boldsymbol{h}_{\mathbf{2}}}$, we use a localized version of the formula of equipartition of energy. We introduce the function $\eta \in W^{1, \infty}(\Omega)$ with the support $\omega$ of the damping a containing strictly the support $\hat{\omega}$ of the function $\boldsymbol{f}$. As a consequence, proceeding as in Step 4 (see also [24]), an approximation $\left(\eta_{j, k}\right)_{j, k}$ of $\eta$ may be defined as follows:

$$
\eta_{j, k}=1 \quad \text { if } \quad(j, k) \in \tilde{I}^{+} \cup \hat{I}^{+}, \quad \eta_{j, k}=0 \quad \text { if } \quad(j, k) \notin G\left(\boldsymbol{\omega}_{\boldsymbol{h}_{1}, \boldsymbol{h}_{\mathbf{2}}}\right) .
$$

Then, multiplying the first equation of (30) by $g(t) \eta_{j, k}^{2} u_{j, k}$ (with $g$ defined in (50)) and proceeding as in Steps 1 and 4 , we obtain (see [24]) using $\hat{\boldsymbol{\omega}}_{\boldsymbol{h}_{1}, \boldsymbol{h}_{\mathbf{2}}} \subset \boldsymbol{\omega}_{\boldsymbol{h}_{1}, \boldsymbol{h}_{2}}$ that:

$$
\begin{aligned}
E_{h_{1}, h_{2}}(0) \leq & C h_{1} h_{2} \sum_{j=0}^{J} \sum_{k \in \mathcal{I}_{j}} \int_{0}^{T} a_{j, k}\left(u_{j, k}^{\prime}(t)\right)^{2} \mathrm{~d} t+C h_{1} h_{2} \sum_{(j, k) \in \boldsymbol{\omega}_{\boldsymbol{h}_{1}, \boldsymbol{h}_{2}}} \int_{0}^{T} g(t)\left(u_{j, k}(t)\right)^{2} \mathrm{~d} t \\
& +C h_{1} h_{2} \sum_{j=0}^{J} \sum_{k \in \mathcal{I}_{j}} \int_{0}^{T}\left\{h_{1}^{2}\left(\frac{u_{j+1, k}^{\prime}-u_{j, k}^{\prime}}{h_{1}}\right)^{2}+h_{2}^{2}\left(\frac{u_{j, k+1}^{\prime}-u_{j, k}^{\prime}}{h_{2}}\right)^{2}\right\} \mathrm{d} t,
\end{aligned}
$$

for any $T>2 \sqrt{2}$. 
Step 6. Conclusion

To conclude the proof it is sufficient to show that there exists a constant $C>0$ such that

$$
\begin{aligned}
\sum_{(j, k) \in \boldsymbol{\omega}_{h_{1}, h_{2}}} & \int_{0}^{T} g(t)\left(u_{j, k}(t)\right)^{2} \mathrm{~d} t \leq C \sum_{j=0}^{J} \sum_{k \in \mathcal{I}_{j}} \int_{0}^{T} a_{j, k}\left(u_{j, k}^{\prime}(t)\right)^{2} \mathrm{~d} t \\
& +C h_{1} h_{2} \sum_{j=0}^{J} \sum_{k \in \mathcal{I}_{j}} \int_{0}^{T}\left\{h_{1}^{2}\left(\frac{u_{j+1, k}^{\prime}-u_{j, k}^{\prime}}{h_{1}}\right)^{2}+h_{2}^{2}\left(\frac{u_{j, k+1}^{\prime}-u_{j, k}^{\prime}}{h_{2}}\right)^{2}\right\} \mathrm{d} t .
\end{aligned}
$$

To do this we may argue by means of a classical compactness uniqueness argument as in Proposition 4.1 in [26]. According to this argument, (57) reduces to an unique continuation property for the solution of the continuous wave equation. But, for that to be true, we need a convergence result guaranteeing that the solutions of the discrete system converge to those of the wave equation. This is done in Theorem 3.1 of Section 3 . Since the analysis described above is analogous to the one developed in [24], we omit the details. This completes the proof of Theorem 2.1 .

\section{Convergence of the numerical scheme}

The aim of this section is to prove a convergence result of the solution of the semi-discrete scheme (21) to the solution of system (1). This allows to conclude that the presence of the viscous terms acts only as a regularization term to damp out the high frequency components and does not change the limit behavior. This will also permit to pass to the limit in equation (28) of Theorem 2.1 to find again the exponential decay of the energy $E$ (Eq. (6)). Before stating the convergence result, let us introduce the two following extensions $P_{h_{1}, h_{2}}$ and $Q_{h_{1}, h_{2}}$ defined by:

$$
\begin{aligned}
P_{h_{1}, h_{2}} \boldsymbol{v}_{\boldsymbol{h}_{\mathbf{1}}, \boldsymbol{h}_{\mathbf{2}}}=\left\{\begin{array}{l}
\text { the continuous function linear in each cell } B_{j, k} \text { such that } \\
P_{h_{1}, h_{2}} \boldsymbol{v}_{\boldsymbol{h}_{\mathbf{1}}, \boldsymbol{h}_{\mathbf{2}}}\left(x_{1, j}, x_{2, k}\right)=v_{j, k}, \\
P_{h_{1}, h_{2}} \boldsymbol{v}_{\boldsymbol{h}_{\mathbf{1}}, \boldsymbol{h}_{\mathbf{2}}}\left(x_{1, j+1}, x_{2, k}\right)=v_{j+1, k}, \\
P_{h_{1}, h_{2}} \boldsymbol{v}_{\boldsymbol{h}_{\mathbf{1}}, \boldsymbol{h}_{\mathbf{2}}}\left(x_{1, j}, x_{2, k+1}\right)=v_{j, k+1}, \\
P_{h_{1}, h_{2}} \boldsymbol{v}_{\boldsymbol{h}_{\mathbf{1}}, \boldsymbol{h}_{\mathbf{2}}}\left(x_{1, j+1}, x_{2, k+1}\right)=v_{j+1, k+1}, \quad j \in[0, J], k \in \mathcal{I}_{j} .
\end{array}\right. \\
Q_{h_{1}, h_{2}} \boldsymbol{v}_{\boldsymbol{h}_{\mathbf{1}}, \boldsymbol{h}_{\mathbf{2}}}=\left\{\begin{array}{l}
\text { the step function defined in each square } \\
\left(\left(j-\frac{1}{2}\right) h_{1},\left(j+\frac{1}{2}\right) h_{1}\right) \times\left(\left(k-\frac{1}{2}\right) h_{2},\left(k+\frac{1}{2}\right) h_{2}\right) \cap \Omega \\
\text { by } Q_{h_{1}, h_{2}} \boldsymbol{v}_{\boldsymbol{h}_{\mathbf{1}}, \boldsymbol{h}_{\mathbf{2}}}(\boldsymbol{x})=v_{j, k}, j \in[0, J], k \in \mathcal{I}_{j} .
\end{array}\right.
\end{aligned}
$$

We now state our convergence result:

Theorem 3.1 (convergence of $\left.\boldsymbol{y}_{\boldsymbol{h}_{1}, \boldsymbol{h}_{\mathbf{2}}}\right)$. Let $\boldsymbol{y}_{\boldsymbol{h}_{1}, \boldsymbol{h}_{2}}$ denote the solution of (21). Assume that $\boldsymbol{a}_{\boldsymbol{h}_{1}, \boldsymbol{h}_{2}},\left(\boldsymbol{y}_{\mathbf{0}}\right)_{\boldsymbol{h}_{1}, \boldsymbol{h}_{2}}$ and $\left(\boldsymbol{y}_{1}\right)_{\boldsymbol{h}_{1}, \boldsymbol{h}_{\mathbf{2}}}$ are such that there is a nonnegative constant $C$ independent of $h_{1}, h_{2}$ with

$$
\left\{\begin{array}{l}
E_{h_{1}, h_{2}}(0) \leq C, \quad Q_{h_{1}, h_{2}} \boldsymbol{a}_{\boldsymbol{h}_{1}, \boldsymbol{h}_{\mathbf{2}}} \rightarrow \text { a weakly } * \text { in } L^{\infty}(\Omega) \\
P_{h_{1}, h_{2}}\left(\boldsymbol{y}_{\mathbf{0}}\right)_{\boldsymbol{h}_{\mathbf{1}}, \boldsymbol{h}_{\mathbf{2}}} \rightarrow y_{0} \text { weakly in } H_{0}^{1}(\Omega), Q_{h_{1}, h_{2}}\left(\boldsymbol{y}_{\mathbf{1}}\right)_{\boldsymbol{h}_{\mathbf{1}}, \boldsymbol{h}_{\mathbf{2}}} \rightarrow y_{1} \text { weakly in } L^{2}(\Omega) .
\end{array}\right.
$$

Then, we have

$$
\left\{\begin{array}{l}
P_{h_{1}, h_{2}} \boldsymbol{y}_{\boldsymbol{h}_{1}, \boldsymbol{h}_{\mathbf{2}}} \rightarrow y \text { weakly* in } L^{\infty}\left(0, \infty ; H_{0}^{1}(\Omega)\right) \\
Q_{h_{1}, h_{2}} \frac{\partial \boldsymbol{y}_{\boldsymbol{h}_{1}, \boldsymbol{h}_{\mathbf{2}}}}{\partial t} \rightarrow \frac{\partial y}{\partial t} \text { weakly } * \text { in } L^{\infty}\left(0, \infty ; L^{2}(\Omega)\right)
\end{array}\right.
$$

where $y$ is the solution of system (1). 
Proof. We decompose the proof in several steps.

Step 1. A priori estimates and weak convergence

Straightforward computations first lead to the two following equalities:

$$
\begin{aligned}
\int_{\boldsymbol{\Omega}_{\boldsymbol{h}_{\mathbf{1}}, \boldsymbol{h}_{\mathbf{2}}} \nabla P_{h_{1}, h_{2}} \boldsymbol{v}_{\boldsymbol{h}_{\mathbf{1}}, \boldsymbol{h}_{\mathbf{2}}}(\boldsymbol{x}) \cdot \nabla} P_{h_{1}, h_{2}} \boldsymbol{w}_{\boldsymbol{h}_{\mathbf{1}}, \boldsymbol{h}_{\mathbf{2}}}(\boldsymbol{x}) \mathrm{d} x \\
=h_{1} h_{2} \sum_{j=0}^{J} \sum_{k \in \mathcal{I}_{j}}\left(\frac{v_{j+1, k}-v_{j, k}}{h_{1}}\right)\left(\frac{w_{j+1, k}-w_{j, k}}{h_{1}}\right) \\
+h_{1} h_{2} \sum_{j=0}^{J} \sum_{k \in \mathcal{I}_{j}}\left(\frac{v_{j, k+1}-v_{j, k}}{h_{2}}\right)\left(\frac{w_{j, k+1}-w_{j, k}}{h_{2}}\right), \\
\int_{\boldsymbol{\Omega}_{\boldsymbol{h}_{\mathbf{1}}, \boldsymbol{h}_{\mathbf{2}}}} Q_{h_{1}, h_{2}} \boldsymbol{v}_{\boldsymbol{h}_{\mathbf{1}}, \boldsymbol{h}_{\mathbf{2}}}(\boldsymbol{x}) Q_{h_{1}, h_{2}} \boldsymbol{w}_{\boldsymbol{h}_{\mathbf{1}}, \boldsymbol{h}_{\mathbf{2}}}(\boldsymbol{x}) \mathrm{d} x=h_{1} h_{2} \sum_{j=0}^{J} \sum_{k \in \mathcal{I}_{j}} v_{j, k} w_{j, k} .
\end{aligned}
$$

Then, according to the definition (24), we get, for every $t \geq 0$,

$$
E_{h_{1}, h_{2}}(t)=\frac{1}{2}\left(\left\|P_{h_{1}, h_{2}} \boldsymbol{y}_{\boldsymbol{h}_{1}, \boldsymbol{h}_{\mathbf{2}}}(t)\right\|_{H_{0}^{1}\left(\boldsymbol{\Omega}_{\left.\boldsymbol{h}_{\mathbf{1}}, \boldsymbol{h}_{\mathbf{2}}\right)}\right)}^{2}+\left\|Q_{h_{1}, h_{2}} \boldsymbol{y}_{\boldsymbol{h}_{\mathbf{1}}, \boldsymbol{h}_{\mathbf{2}}}^{\prime}(t)\right\|_{L^{2}\left(\boldsymbol{\Omega}_{\boldsymbol{h}_{\mathbf{1}}, \boldsymbol{h}_{\mathbf{2}}}\right)}^{2}\right) .
$$

Then, thanks to (60), (63) and the decreasing character of $E_{h_{1}, h_{2}}$, we deduce that $P_{h_{1}, h_{2}} \boldsymbol{y}_{\boldsymbol{h}_{1}, \boldsymbol{h}_{\mathbf{2}}}$ is bounded in $L^{\infty}\left(0, \infty ; H_{0}^{1}\left(\boldsymbol{\Omega}_{\boldsymbol{h}_{\mathbf{1}}, \boldsymbol{h}_{\mathbf{2}}}\right)\right) \cap W^{1, \infty}\left(0, \infty ; L^{2}\left(\boldsymbol{\Omega}_{\boldsymbol{h}_{\mathbf{1}}, \boldsymbol{h}_{\mathbf{2}}}\right)\right)$, while $Q_{h_{1}, h_{2}} \boldsymbol{y}_{\boldsymbol{h}_{\mathbf{1}}, \boldsymbol{h}_{\mathbf{2}}}^{\prime}$ is bounded in $L^{\infty}\left(0, \infty ; L^{2}\left(\boldsymbol{\Omega}_{\boldsymbol{h}_{\mathbf{1}}, \boldsymbol{h}_{\mathbf{2}}}\right)\right)$. Then, since

$$
\left\|Q_{h_{1}, h_{2}} \boldsymbol{y}_{\boldsymbol{h}_{\mathbf{1}}, \boldsymbol{h}_{\mathbf{2}}}\right\|_{L^{2}\left(\boldsymbol{\Omega}_{\boldsymbol{h}_{1}, h_{\mathbf{2}}}\right)} \leq\left\|\left(P_{h_{1}, h_{2}}-Q_{h_{1}, h_{2}}\right) \boldsymbol{y}_{\boldsymbol{h}_{1}, \boldsymbol{h}_{\mathbf{2}}}\right\|_{L^{2}\left(\boldsymbol{\Omega}_{\boldsymbol{h}_{\mathbf{1}}, \boldsymbol{h}_{\mathbf{2}}}\right)}+\left\|P_{h_{1}, h_{2}} \boldsymbol{y}_{\boldsymbol{h}_{1}, \boldsymbol{h}_{\mathbf{2}}}\right\|_{L^{2}\left(\boldsymbol{\Omega}_{\boldsymbol{h}_{1}, \boldsymbol{h}_{\mathbf{2}}}\right)}
$$

and $E_{h_{1}, h_{2}}$ is bounded, straightforward computations leads to

$$
\left\|P_{h_{1}, h_{2}} \boldsymbol{y}_{\boldsymbol{h}_{\mathbf{1}}, \boldsymbol{h}_{\mathbf{2}}}-Q_{h_{1}, h_{2}} \boldsymbol{y}_{\boldsymbol{h}_{1}, \boldsymbol{h}_{\mathbf{2}}}\right\|_{L^{2}\left(\boldsymbol{\Omega}_{\boldsymbol{h}_{\mathbf{1}}, \boldsymbol{h}_{\mathbf{2}}}\right)}=O\left(h_{1} h_{2}\right), \forall t \geq 0
$$

what allows to deduce that $Q_{h_{1}, h_{2}} \boldsymbol{y}_{\boldsymbol{h}_{\mathbf{1}}, \boldsymbol{h}_{\mathbf{2}}}$ is bounded in $L^{\infty}\left(0, \infty ; L^{2}\left(\boldsymbol{\Omega}_{\boldsymbol{h}_{1}, \boldsymbol{h}_{\mathbf{2}}}\right)\right)$. In the same way, multiplying the first equation of $(21)$ by $y_{j, k}$, integrating by part over $[0, T]$ and rearranging the index, one can also show that $P_{h_{1}, h_{2}} \boldsymbol{y}_{\boldsymbol{h}_{1}, \boldsymbol{h}_{\mathbf{2}}}^{\prime}$ is bounded in $L^{\infty}\left(0, \infty ; L^{2}\left(\boldsymbol{\Omega}_{\boldsymbol{h}_{\mathbf{1}}, \boldsymbol{h}_{\mathbf{2}}}\right)\right)$ such that $P_{h_{1}, h_{2}} \boldsymbol{y}_{\boldsymbol{h}_{\mathbf{1}}, \boldsymbol{h}_{\mathbf{2}}}$ is also bounded in $W^{1, \infty}\left(0, \infty ; L^{2}\left(\boldsymbol{\Omega}_{\boldsymbol{h}_{1}, \boldsymbol{h}_{\mathbf{2}}}\right)\right)$.

Furthermore, taking into account that solutions both in the continuous and the semi-discrete case satisfy Dirichlet boundary conditions, we can extend them by zero to the whole unit box $B$. The extension operator is denoted by the symbol $\sim$. Consequently, we can consider each $\boldsymbol{y}_{\boldsymbol{h}_{1}, \boldsymbol{h}_{\mathbf{2}}}$, as well as the solution of (1), as solution of $H_{0}^{1}(B)$, and therefore the boundedness of $E_{h_{1}, h_{2}}(t)$ mentioned above still holds in the domain $B$, independent of $h_{1}$ and $h_{2}$. Thus, we can extract subsequences (that we still denote by $\tilde{\boldsymbol{y}}_{\boldsymbol{h}_{1}, \boldsymbol{h}_{\mathbf{2}}}$ ), such that

$$
\left\{\begin{array}{l}
P_{h_{1}, h_{2}} \tilde{\boldsymbol{y}}_{\boldsymbol{h}_{1}, \boldsymbol{h}_{\mathbf{2}}} \rightarrow \tilde{y} \quad \text { weakly } * \text { in } L^{\infty}\left(0, \infty ; H_{0}^{1}(B)\right), \\
P_{h_{1}, h_{2}} \tilde{\boldsymbol{y}}_{\boldsymbol{h}_{\mathbf{1}}, \boldsymbol{h}_{\mathbf{2}}}^{\prime} \rightarrow \tilde{y}^{\prime} \quad \text { weakly } * \text { in } L^{\infty}\left(0, \infty ; L^{2}(B)\right), \\
P_{h_{1}, h_{2}} \tilde{\boldsymbol{y}}_{\boldsymbol{h}_{\mathbf{1}}, \boldsymbol{h}_{\mathbf{2}}} \rightarrow \tilde{y} \quad \text { strongly in } L_{l o c}^{2}\left(0, \infty ; L^{2}(B)\right), \\
Q_{h_{1}, h_{2}} \tilde{\boldsymbol{y}}_{\boldsymbol{h}_{\mathbf{1}}, \boldsymbol{h}_{\mathbf{2}}} \rightarrow \tilde{y} \quad \text { weakly } * \text { in } L^{\infty}\left(0, \infty ; L^{2}(B)\right), \\
Q_{h_{1}, h_{2}} \tilde{\boldsymbol{y}}_{\boldsymbol{h}_{\mathbf{1}}, \boldsymbol{h}_{\mathbf{2}}}^{\prime} \rightarrow \tilde{y}^{\prime} \quad \text { weakly } * \text { in } L^{\infty}\left(0, \infty ; L^{2}(B)\right), \\
h_{i} P_{h_{1}, h_{2}} \tilde{\boldsymbol{y}}_{\boldsymbol{h}_{\mathbf{1}}, \boldsymbol{h}_{\mathbf{2}}}^{\prime} \rightarrow 0 \quad \text { weakly } * \text { in } L^{2}\left(0, \infty ; H_{0}^{1}(B)\right), i=1,2 .
\end{array}\right.
$$

The last convergence in (66) follows from the second one and the boundness of the sequence $\left\{h_{i} P_{h_{1}, h_{2}}\right\}, i=1,2$, in the space $L^{2}\left(0, \infty ; H_{0}^{1}(B)\right)$ easily obtained by multiplying the first equation of (21) by $\tilde{y}_{j, k}^{\prime}$, integration by parts and rearranging the index. 
Note that in (66), we implicit claim that the limits of $P_{h_{1}, h_{2}} \tilde{\boldsymbol{y}}_{\boldsymbol{h}_{1}, \boldsymbol{h}_{\mathbf{2}}}$ and $Q_{h_{1}, h_{2}} \tilde{\boldsymbol{y}}_{\boldsymbol{h}_{1}, \boldsymbol{h}_{\mathbf{2}}}$ are the same which is a consequence of $(65)$.

Step 2. Characterization of the limit $\tilde{y}$

Let us now show that the limit $\tilde{y}$ is the solution of $(1)$. To this end, let $w \in \mathcal{D}(\Omega \times(0, \infty))$ and extend it by zero to the whole $B$. We set $\tilde{\boldsymbol{w}}_{\boldsymbol{h}_{\mathbf{1}}, \boldsymbol{h}_{\mathbf{2}}}=\left(\tilde{w}_{j, k}\right)_{j, k}$ where $\tilde{w}_{j, k}=\tilde{w}\left(x_{1, j}, x_{2, k}\right)$. Multiplying the first equation of $(21)$ by $\tilde{w}_{j, k}$, integrating by parts in $(0, \infty)$ and taking the sum, we find

$$
\begin{aligned}
& \sum_{j=0}^{J} \sum_{k=0}^{K} \int_{0}^{\infty} \tilde{y}_{j, k} \tilde{w}_{j, k}^{\prime \prime} \mathrm{d} t-\sum_{j=0}^{J} \sum_{k=0}^{K} \int_{0}^{\infty} \tilde{a}_{j, k} \tilde{y}_{j, k} \tilde{w}_{j, k}^{\prime} \mathrm{d} t \\
& +\sum_{j=0}^{J} \sum_{k=0}^{K} \int_{0}^{\infty}\left[\left(\frac{\tilde{y}_{j+1, k}-\tilde{y}_{j, k}}{h_{1}}\right)\left(\frac{\tilde{w}_{j+1, k}-\tilde{w}_{j, k}}{h_{1}}\right)+\left(\frac{\tilde{y}_{j, k+1}-\tilde{y}_{j, k}}{h_{2}}\right)\left(\frac{\tilde{w}_{j, k+1}-\tilde{w}_{j, k}}{h_{2}}\right)\right] \mathrm{d} t \\
& +\sum_{j=0}^{J} \sum_{k=0}^{K} \int_{0}^{\infty}\left[h_{1}^{2}\left(\frac{\tilde{y}_{j+1, k}^{\prime}-\tilde{y}_{j, k}^{\prime}}{h_{1}}\right)\left(\frac{\tilde{w}_{j+1, k}-\tilde{w}_{j, k}}{h_{1}}\right)\right. \\
& \left.+h_{2}^{2}\left(\frac{\tilde{y}_{j, k+1}^{\prime}-\tilde{y}_{j, k}^{\prime}}{h_{2}}\right)\left(\frac{\tilde{w}_{j, k+1}-\tilde{w}_{j, k}}{h_{2}}\right)\right] \mathrm{d} t=0 .
\end{aligned}
$$

Using the definitions of $P_{h_{1}, h_{2}}$ and $Q_{h_{1}, h_{2}}$, we can check that (67) is equivalent to

$$
\begin{aligned}
\int_{0}^{\infty} & \int_{B} Q_{h_{1}, h_{2}} \tilde{\boldsymbol{y}}_{\boldsymbol{h}_{\mathbf{1}}, \boldsymbol{h}_{\mathbf{2}}} Q_{h_{1}, h_{2}} \tilde{\boldsymbol{w}}_{\boldsymbol{h}_{\mathbf{1}}, \boldsymbol{h}_{\mathbf{2}}}^{\prime \prime} \mathrm{d} x \mathrm{~d} t \\
& +\int_{0}^{\infty} \int_{B}^{\infty} \nabla P_{h_{1}, h_{2}} \tilde{\boldsymbol{y}}_{\boldsymbol{h}_{\mathbf{1}}, \boldsymbol{h}_{\mathbf{2}}} \cdot \nabla P_{h_{1}, h_{2}} \tilde{\boldsymbol{w}}_{\boldsymbol{h}_{\mathbf{1}}, \boldsymbol{h}_{\mathbf{2}}} \mathrm{d} x \mathrm{~d} t \\
& -\int_{0}^{\infty} \int_{B_{J}} Q_{h_{1}, h_{2}} \tilde{\boldsymbol{y}}_{\boldsymbol{h}_{\mathbf{1}}, h_{\mathbf{2}}} Q_{h_{1}, h_{2}} \tilde{\boldsymbol{w}}_{\boldsymbol{h}_{\mathbf{1}}, \boldsymbol{h}_{\mathbf{2}}}^{\prime} Q_{h_{1}, h_{2}} \tilde{\boldsymbol{a}}_{\boldsymbol{h}_{1}, h_{\mathbf{2}}} \mathrm{d} x \mathrm{~d} t \\
& +h_{1} h_{2} \sum_{j=0}^{K} \sum_{k=0}^{\infty} \int_{0}^{\infty}\left[h_{1}^{2}\left(\frac{\tilde{y}_{j+1, k}^{\prime}-\tilde{y}_{j, k}^{\prime}}{h_{1}}\right)\left(\frac{\tilde{w}_{j+1, k}-\tilde{w}_{j, k}}{h_{1}}\right)\right. \\
& \left.+h_{2}^{2}\left(\frac{\tilde{y}_{j, k+1}^{\prime}-\tilde{y}_{j, k}^{\prime}}{h_{2}}\right)\left(\frac{\tilde{w}_{j, k+1}-\tilde{w}_{j, k}}{h_{2}}\right)\right] \mathrm{d} t=0 .
\end{aligned}
$$

Now, we observe that, if we show that for every $w \in \mathcal{D}(\Omega \times(0, \infty))$

$$
\left\{\begin{array}{l}
P_{h_{1}, h_{2}} \tilde{\boldsymbol{w}}_{\boldsymbol{h}_{\mathbf{1}}, \boldsymbol{h}_{\mathbf{2}}} \rightarrow \tilde{w} \text { strongly in } L^{2}\left(0, \infty ; H_{0}^{1}(B)\right), \\
Q_{h_{1}, h_{2}} \tilde{\boldsymbol{w}}_{\boldsymbol{h}_{\mathbf{1}}, \boldsymbol{h}_{\mathbf{2}}} \rightarrow \tilde{w} \text { strongly in } L^{2}\left(0, \infty ; L^{2}(B)\right)
\end{array}\right.
$$

then, we will be able to pass to the limit in (68) leading with (66) to

$$
\int_{0}^{\infty} \int_{B \cap \operatorname{supp} w} \tilde{y} w^{\prime \prime} \mathrm{d} x \mathrm{~d} t+\int_{0}^{\infty} \int_{B \cap \operatorname{supp} w} \nabla \tilde{y} \cdot \nabla w \mathrm{~d} x \mathrm{~d} t-\int_{0}^{\infty} \int_{B \cap \operatorname{supp} w} \tilde{y} w^{\prime} a \mathrm{~d} x \mathrm{~d} t=0
$$

where supp $w$ denotes the support of $w \in \mathcal{D}(\Omega \times(0, \infty))$, what guaranties that

$$
\int_{0}^{\infty} \int_{\Omega} \tilde{y} w^{\prime \prime} \mathrm{d} x \mathrm{~d} t+\int_{0}^{\infty} \int_{\Omega} \nabla \tilde{y} \cdot \nabla w \mathrm{~d} x \mathrm{~d} t-\int_{0}^{\infty} \int_{\Omega} \tilde{y} w^{\prime} a \mathrm{~d} x \mathrm{~d} t=0
$$

for all $w \in \mathcal{D}(\Omega \times(0, \infty))$. 
Step 3. Property of $\tilde{y}(t)$ for all $t \geq 0$

Let us first show that $\tilde{y}(t) \in H_{0}^{1}(\Omega)$ for all $t \geq 0$. Since $P_{h_{1}, h_{2}} \boldsymbol{y}_{\boldsymbol{h}_{1}, \boldsymbol{h}_{2}}$ belongs to $H_{0}^{1}\left(\boldsymbol{\Omega}_{\boldsymbol{h}_{1}, \boldsymbol{h}_{\mathbf{2}}}\right) \subset H_{0}^{1}(B)$, the limit $\tilde{y}(t)$ belongs to $H_{0}^{1}(B)$. Furthermore, since $\partial \Omega$ is Lipschitz by assumption and also $\partial \boldsymbol{\Omega}_{\boldsymbol{h}_{1}, \boldsymbol{h}_{2}}$ by construction, it possess the so-called $\epsilon$-cone property, introduced in [4]. This implies that the bounded sequence $\left(\boldsymbol{\Omega}_{\boldsymbol{h}_{1}, \boldsymbol{h}_{2}}\right)_{h_{1}, h_{2}}$ defined, for each $h_{1}, h_{2}$ fixed, as the largest domain included in $\Omega$ and union of cell $B_{j, k}$ converges towards $\Omega$ with respect to the complementary-Hausdorff topology (see [20]). This property implies, in particular, that the limit of the sequence $P_{h_{1}, h_{2}} \boldsymbol{y}_{\boldsymbol{h}_{1}, \boldsymbol{h}_{2}}$ defined on $\boldsymbol{\Omega}_{\boldsymbol{h}_{1}, \boldsymbol{h}_{2}}$ extended to zero on $B \backslash \Omega$ belongs to $H_{0}^{1}(\Omega)$ for any time $t \geq 0$. Actually, in our situation - $\left(\boldsymbol{\Omega}_{\boldsymbol{h}_{1}, \boldsymbol{h}_{2}}\right)$ is an increasing sequence include in $\Omega$ - we could obtain the same property using more classical results of spectral analysis (see [10] and the references therein).

Let us now show that $\tilde{y}(0)=y_{0}$ and $\tilde{y}^{\prime}(0)=y_{1}$. For this purpose, let $v \in \mathcal{D}(\Omega)$ and $l \in \mathcal{D}([0, \infty))$. Then, multiplying the first equation of (21) by $v_{j, k} l$, integrating by parts on $[0, \infty)$ and taking the sum, we find

$$
\begin{aligned}
& -\sum_{j=0}^{J} \sum_{k=0}^{K}\left(y_{1}\right)_{j, k} \tilde{v}_{j, k} l(0)+\sum_{j=0}^{J} \sum_{k=0}^{K}\left(y_{0}\right)_{j, k} \tilde{v}_{j, k} l^{\prime}(0)-\sum_{j=0}^{J} \sum_{k=0}^{K} \tilde{a}_{j, k}\left(y_{0}\right)_{j, k} \tilde{v}_{j, k} l(0) \\
& +\sum_{j=0}^{J} \sum_{k=0}^{K} \int_{0}^{\infty} \tilde{y}_{j, k} \tilde{v}_{j, k} l^{\prime \prime} \mathrm{d} t-\sum_{j=0}^{J} \sum_{k=0}^{K} \int_{0}^{\infty} \tilde{a}_{j, k} \tilde{y}_{j, k} \tilde{v}_{j, k} l^{\prime} \mathrm{d} t \\
& +\sum_{j=0}^{J} \sum_{k=0}^{K} \int_{0}^{\infty}\left[\left(\frac{\tilde{y}_{j+1, k}-\tilde{y}_{j, k}}{h_{1}}\right)\left(\frac{\tilde{v}_{j+1, k}-\tilde{v}_{j, k}}{h_{1}}\right)+\left(\frac{\tilde{y}_{j, k+1}-\tilde{y}_{j, k}}{h_{2}}\right)\left(\frac{\tilde{v}_{j, k+1}-\tilde{v}_{j, k}}{h_{2}}\right)\right] l \mathrm{~d} t \\
& +\sum_{j=0}^{J} \sum_{k=0}^{K} \int_{0}^{\infty}\left[h_{1}^{2}\left(\frac{\tilde{y}_{j+1, k}^{\prime}-\tilde{y}_{j, k}^{\prime}}{h_{1}}\right)\left(\frac{\tilde{v}_{j+1, k}-\tilde{v}_{j, k}}{h_{1}}\right)\right. \\
& \left.+h_{2}^{2}\left(\frac{\tilde{y}_{j, k+1}^{\prime}-\tilde{y}_{j, k}^{\prime}}{h_{2}}\right)\left(\frac{\tilde{v}_{j, k+1}-\tilde{v}_{j, k}}{h_{2}}\right)\right] l \mathrm{~d} t=0 .
\end{aligned}
$$

Using the definitions of $P_{h_{1}, h_{2}}$ and $Q_{h_{1}, h_{2}}$ and proceeding as in the last steps, we can check that (72) is equivalent to

$$
\begin{aligned}
& -l(0) \int_{B} Q_{h_{1}, h_{1}}\left(\boldsymbol{y}_{1}\right)_{\boldsymbol{h}_{1}, h_{2}} Q_{h_{1}, h_{2}} \tilde{\boldsymbol{v}}_{\boldsymbol{h}_{1}, \boldsymbol{h}_{\mathbf{2}}} \mathrm{d} x+l^{\prime}(0) \int_{B} Q_{h_{1}, h_{2}}\left(\boldsymbol{y}_{\mathbf{0}}\right)_{h_{1}, \boldsymbol{h}_{\mathbf{2}}} Q_{h_{1}, h_{2}} \tilde{\boldsymbol{v}}_{\boldsymbol{h}_{\mathbf{1}}, \boldsymbol{h}_{\mathbf{2}}} \mathrm{d} x \\
& -l(0) \int_{B} Q_{h_{1}, h_{2}}\left(\boldsymbol{y}_{\mathbf{0}}\right)_{\boldsymbol{h}_{\mathbf{1}}, h_{\mathbf{2}}} Q_{h_{1}, h_{2}} \tilde{\boldsymbol{v}}_{\boldsymbol{h}_{1}, h_{\mathbf{2}}} Q_{h_{1}, h_{2}} \tilde{\boldsymbol{a}}_{\boldsymbol{h}_{1}, h_{\mathbf{2}}} \mathrm{d} x \\
& +\int_{0}^{\infty} \int_{B} Q_{h_{1}, h_{2}} \tilde{\boldsymbol{y}}_{\boldsymbol{h}_{1}, \boldsymbol{h}_{2}} Q_{h_{1}, h_{2}} \tilde{\boldsymbol{v}}_{\boldsymbol{h}_{1}, \boldsymbol{h}_{2}} l^{\prime \prime} \mathrm{d} x \mathrm{~d} t \\
& +\int_{0}^{\infty} \int_{B} \nabla P_{h_{1}, h_{2}} \tilde{\boldsymbol{y}}_{h_{1}, h_{2}} \cdot \nabla P_{h_{1}, h_{2}} \tilde{\boldsymbol{v}}_{h_{1}, h_{2}} l \mathrm{~d} x \mathrm{~d} t \\
& -\int_{0}^{\infty} \int_{B} Q_{h_{1}, h_{2}} \tilde{\boldsymbol{y}}_{\boldsymbol{h}_{1}, \boldsymbol{h}_{2}} Q_{h_{1}, h_{2}} \tilde{\boldsymbol{v}}_{\boldsymbol{h}_{1}, \boldsymbol{h}_{2}} Q_{h_{1}, h_{2}} \tilde{\boldsymbol{a}}_{\boldsymbol{h}_{1}, \boldsymbol{h}_{2}} l^{\prime} \mathrm{d} x \mathrm{~d} t \\
& +h_{1} h_{2} \sum_{j=0}^{J} \sum_{k=0}^{K} \int_{0}^{\infty}\left[h_{1}^{2}\left(\frac{\tilde{y}_{j+1, k}^{\prime}-\tilde{y}_{j, k}^{\prime}}{h_{1}}\right)\left(\frac{\tilde{v}_{j+1, k}-\tilde{v}_{j, k}}{h_{1}}\right)\right. \\
& \left.+h_{2}^{2}\left(\frac{\tilde{y}_{j, k+1}^{\prime}-\tilde{y}_{j, k}^{\prime}}{h_{2}}\right)\left(\frac{\tilde{v}_{j, k+1}-\tilde{v}_{j, k}}{h_{2}}\right)\right] l \mathrm{~d} t=0 .
\end{aligned}
$$


Passing to the limit as $\left(h_{1}, h_{2}\right) \rightarrow(0,0)$ in $(73)$, we get

$$
\begin{aligned}
-l(0) \int_{\Omega} y_{1} v \mathrm{~d} x+l^{\prime}(0) \int_{\Omega} y_{0} v \mathrm{~d} x-l(0) & \int_{\Omega} y_{0} v a \mathrm{~d} x \\
& +\int_{0}^{\infty} \int_{\Omega} y v l^{\prime \prime} \mathrm{d} x \mathrm{~d} t+\int_{0}^{\infty} \int_{\Omega} \nabla y \cdot \nabla v l \mathrm{~d} x \mathrm{~d} t-\int_{0}^{\infty} \int_{B} y v a l^{\prime} \mathrm{d} x \mathrm{~d} t=0
\end{aligned}
$$

from which we derive $\tilde{y}(0)=y_{0}$ and $\tilde{y}^{\prime}(0)=y_{1}$. Since system (1) has a unique solution, we conclude that the convergence results in (66) hold for the whole sequence $\left\{\left(h_{1}, h_{2}\right)\right\}$.

\section{Step 4. Proof of (69)}

To conclude the proof, let us prove (69). First observe that, for all $w \in \mathcal{D}(\Omega \times(0, \infty))$, we have

$$
\begin{aligned}
& \int_{0}^{\infty} \int_{B_{J}}\left|\nabla\left(P_{h_{1}, h_{2}} \tilde{\boldsymbol{w}}_{h_{1}, h_{\mathbf{2}}}\right)-\nabla \tilde{w}\right|^{2} \mathrm{~d} x \mathrm{~d} t= \\
& h_{1} h_{2} \sum_{j=0}^{K} \sum_{k=0}^{K} \int_{0}^{\infty}\left[\left(\frac{\tilde{w}_{j+1, k}-\tilde{w}_{j, k}}{h_{1}}\right)^{2}+\left(\frac{\tilde{w}_{j, k+1}-\tilde{w}_{j, k}}{h_{2}}\right)^{2}\right] \mathrm{d} t+\int_{0}^{\infty} \int_{B}|\nabla \tilde{w}|^{2} \mathrm{~d} x \mathrm{~d} t \\
& -2 h_{1} h_{2} \sum_{j=0}^{J} \sum_{k=0}^{K} \int_{0}^{\infty}\left\{\left(\frac{\tilde{w}_{j+1, k}-\tilde{w}_{j, k}}{h_{1}}\right) \int_{0}^{1} \int_{0}^{1} \frac{\partial \tilde{w}}{\partial x_{1}}\left(\left(j+s_{1}\right) h_{1}, x_{2}\right) \mathrm{d} s_{1} \mathrm{~d} x_{2}\right. \\
& \left.+\left(\frac{\tilde{w}_{j, k+1}-\tilde{w}_{j, k}}{h_{2}}\right) \int_{0}^{1} \int_{0}^{1} \frac{\partial \tilde{w}}{\partial x_{2}}\left(x_{1},\left(k+s_{2}\right) h_{2}\right) \mathrm{d} s_{2} \mathrm{~d} x_{1}\right\} \mathrm{d} t, \text { where } s=\left(s_{1}, s_{2}\right),
\end{aligned}
$$

so that using the properties of Riemann sums, it follows that

$$
\lim _{\left(h_{1}, h_{2}\right) \rightarrow 0} \int_{0}^{\infty} \int_{\Omega}\left|\nabla\left(P_{h_{1}, h_{2}} \boldsymbol{w}_{\boldsymbol{h}_{\mathbf{1}}, \boldsymbol{h}_{\mathbf{2}}}\right)-\nabla w\right|^{2} \mathrm{~d} x \mathrm{~d} t=0,
$$

leading to the first convergence in (69). The second one is obtained using the same argument and with

$$
\begin{aligned}
\int_{0}^{\infty} \int_{B}\left(Q_{h_{1}, h_{2}} \tilde{\boldsymbol{w}}_{h_{1}, h_{\mathbf{2}}}-\tilde{w}\right)^{2} \mathrm{~d} x \mathrm{~d} t=h_{1} h_{2} \sum_{j=0}^{J} \sum_{k=0}^{K} \int_{0}^{\infty}\left|\tilde{w}\left(j h_{1}, k h_{2}, t\right)\right|^{2} \mathrm{~d} t+\int_{0}^{\infty} \int_{B} \tilde{w}^{2} \mathrm{~d} x \mathrm{~d} t \\
-2 h_{1} h_{2} \sum_{j=0}^{J} \sum_{k=0}^{K} \int_{0}^{\infty}\left\{\int_{0}^{\frac{1}{2}} \int_{0}^{\frac{1}{2}} \tilde{w}_{j, k} \tilde{w}\left(\left(j+s_{1}\right) h_{1},\left(k+s_{2}\right) h_{2}\right) \mathrm{d} s_{1} \mathrm{~d} s_{2}\right. \\
+\int_{0}^{\frac{1}{2}} \int_{\frac{1}{2}}^{1} \tilde{w}_{j+1, k} \tilde{w}\left(\left(j+s_{1}\right) h_{1},\left(k+s_{2}\right) h_{2}\right) \mathrm{d} s_{1} \mathrm{~d} s_{2} \\
+\int_{\frac{1}{2}}^{1} \int_{0}^{\frac{1}{2}} \tilde{w}_{j, k+1} \tilde{w}\left(\left(j+s_{1}\right) h_{1},\left(k+s_{2}\right) h_{2}\right) \mathrm{d} s_{1} \mathrm{~d} s_{2} \\
\left.+\int_{\frac{1}{2}}^{1} \int_{\frac{1}{2}}^{1} \tilde{w}_{j+1, k+1} \tilde{w}\left(\left(j+s_{1}\right) h_{1},\left(k+s_{2}\right) h_{2}\right) \mathrm{d} s_{1} \mathrm{~d} s_{2} \cdot\right\} \mathrm{d} t .
\end{aligned}
$$

Again, using the properties of Riemann sums, we obtain the second convergence in (69). This concludes the proof of Theorem 3.1 .

Remark 3.2. Assuming strong convergence of the initial data instead of (60), one may also show that convergence (61) holds for a more strong topology (see for instance [15], Chap. 4) leading to

$$
E_{h_{1}, h_{2}}(t) \rightarrow E(t), \quad \forall t \geq 0, \quad \text { when } \quad\left(h_{1}, h_{2}\right) \rightarrow(0,0) .
$$


Precisely, let us show that (78) holds if we assume that the initial data satisfies

$$
E_{h_{1}, h_{2}}(0) \rightarrow E(0) \text { as }\left(h_{1}, h_{2}\right) \rightarrow(0,0) .
$$

Since $E_{h_{1}, h_{2}}(t)$ decreases to zero as $t \rightarrow \infty$, it follows from (25) that

$$
E_{h_{1}, h_{2}}(0)=h_{1} h_{2} \sum_{j=0}^{J} \sum_{k \in I_{j}} \int_{0}^{\infty} a_{j, k}\left(y_{j, k}^{\prime}\right)^{2} \mathrm{~d} t+h_{1} h_{2} \sum_{j=0}^{J} \sum_{k \in I_{j}} \int_{0}^{\infty}\left\{h_{1}^{2}\left(\frac{y_{j+1, k}^{\prime}-y_{j, k}^{\prime}}{h_{1}}\right)^{2}+h_{2}^{2}\left(\frac{y_{j+1, k}^{\prime}-y_{j, k}^{\prime}}{h_{1}}\right)^{2}\right\} \mathrm{d} t .
$$

On the other hand, being $\Omega$ of $\mathcal{C}^{2}$ class, $E(t) \rightarrow 0$ as $t \rightarrow \infty$, and therefore

$$
E(0)=\int_{0}^{\infty} \int_{\Omega} a(\boldsymbol{x})\left|y_{t}\right|^{2} \mathrm{~d} x \mathrm{~d} t
$$

Thus, from (79)-(81) it follows that

$$
\begin{aligned}
\limsup _{\left(h_{1}, h_{2}\right) \rightarrow(0,0)} h_{1} h_{2} \sum_{j=0}^{J} \sum_{k \in I_{j}} \int_{0}^{\infty} a_{j, k}\left(y_{j, k}^{\prime}\right)^{2} \mathrm{~d} t & =\limsup _{\left(h_{1}, h_{2}\right) \rightarrow(0,0)} \int_{0}^{\infty} \int_{\Omega} Q_{h_{1}, h_{2}} a_{h_{1}, h_{2}}\left|Q_{h, h_{2}} y_{h_{1}, h_{2}}^{\prime}\right|^{2} \mathrm{~d} x \mathrm{~d} t \\
& \leq \int_{0}^{\infty} \int_{\Omega} a(\boldsymbol{x})\left|y_{t}\right|^{2} \mathrm{~d} x \mathrm{~d} t,
\end{aligned}
$$

which combined with the weak convergence proved in Theorem 3.1 yields

$$
h_{1} h_{2} \sum_{j=0}^{J} \sum_{k \in I_{j}} a_{j, k}\left(y_{j, k}^{\prime}\right)^{2} \rightarrow \int_{\Omega} a(\boldsymbol{x})\left|y_{t}\right|^{2} \mathrm{~d} x \text { strongly in } L^{2}(0, \infty) .
$$

Consequently,

$$
h_{i} P_{h_{1}, h_{2}} y_{h_{1}, h_{2}}^{\prime} \rightarrow 0 \text { strongly in } L^{2}\left(0,+\infty ; H_{0}^{1}(\Omega)\right), \quad i=1,2 \text {. }
$$

Moreover, thanks to

$$
E(0)=E(t)+\int_{0}^{t} \int_{\Omega} a(\boldsymbol{x})\left|y_{t}\right|^{2} \mathrm{~d} x \mathrm{~d} t, \forall t>0
$$

we have

$$
\begin{aligned}
\left|E_{h_{1}, h_{2}}(t)-E(t)\right| \leq & \left|E_{h_{1}, h_{2}}(0)-E(0)\right|+h_{1} h_{2} \int_{0}^{t} \int_{\Omega}\left|\nabla P_{h_{1}, h_{2}} y_{h_{1}, h_{2}}^{\prime}\right|^{2} \mathrm{~d} x \mathrm{~d} t \\
& +\left.\left|h_{1} h_{2} \sum_{j=0} \sum_{k \in I_{j}} \int_{0}^{t} a_{j, k}\left(y_{j, k}^{\prime}\right)^{2} \mathrm{~d} t-\int_{0}^{t} \int_{\Omega} a(\boldsymbol{x})\right| y_{t}\right|^{2} \mathrm{~d} x \mathrm{~d} t \mid \\
\leq & \left|E_{h_{1}, h_{2}}(0)-E(0)\right|+h_{1} h_{2} \int_{0}^{\infty} \int_{\Omega}\left|\nabla P_{h_{1}, h_{2}} y_{h_{1}, h_{2}}^{\prime}\right|^{2} \mathrm{~d} x \mathrm{~d} t \\
& +\left.\int_{0}^{t}\left|h_{1} h_{2} \sum_{j=0}^{J} \sum_{k \in I_{j}} a_{j, k}\left(y_{j, k}^{\prime}\right)^{2}-\int_{\Omega} a(\boldsymbol{x})\right| y_{t}\right|^{2} \mathrm{~d} x \mid \mathrm{d} t \\
\leq & \left|E_{h_{1}, h_{2}}(0)-E(0)\right|+h_{1} h_{2} \int_{0}^{\infty} \int_{\Omega}\left|\nabla P_{h_{1}, h_{2}} y_{h_{1}, h_{2}}^{\prime}\right|^{2} \mathrm{~d} x \mathrm{~d} t \\
& +\left.\int_{0}^{\infty}\left|h_{1} h_{2} \sum_{j=0}^{J} \sum_{k \in I_{j}} a_{j, k}\left(y_{j, k}^{\prime}\right)^{2}-\int_{\Omega} a(\boldsymbol{x})\right| y_{t}\right|^{2} \mathrm{~d} x \mid \mathrm{d} t
\end{aligned}
$$

and (78) follows. 


\section{Numerical EXPERIMENTS}

The aim of this section is to present some simple numerical experiments in order to confirm the theoretical results that indicate the need and efficiency of adding an extra viscous damping term. This is done over a fully-discrete approximation.

A rigorous analysis of the results in this paper to the fully discrete case remains to be done. However, the recent results in $[17,19]$ on the discrete Ingham inequality and its applications to fully-discrete approximation of the 1-D wave equation suggest that, very likely, the viscous term will suffice to guarantee the uniform decay property to hold in this case too.

\subsection{Description of a fully discrete finite-difference scheme}

We describe a fully discrete - in space and also in time - scheme associated to system (27). In order to simplify the formulation, we assume now that $h_{1}=h_{2} \equiv h$. Let us denote by $y_{j, k}^{n}$ the approximation of the solution $y$ of $(27)$ at the point of coordinates $\left(x_{1, j}, x_{2, k}\right)$ and at time $n \Delta t$ :

$$
y_{j, k}^{n} \approx y\left(x_{1, j}, x_{2, k}, n \Delta t\right) .
$$

Here and in the sequel $\Delta t$ denotes the time-step and $n$ a nonnegative integer in the set $\{0, N\} . N$ and $\Delta t$ are defined such that $T=N \Delta t$. Therefore, we consider the following finite-difference approximation for the time derivative:

$$
\frac{\partial^{2} y}{\partial t^{2}}(\boldsymbol{x}, t) \approx \frac{y(\boldsymbol{x}, t+\Delta t)-2 y(\boldsymbol{x}, t)+y(\boldsymbol{x}, t-\Delta t)}{(\Delta t)^{2}}, \quad \frac{\partial y}{\partial t}(\boldsymbol{x}, t) \approx \frac{y(\boldsymbol{x}, t+\Delta t)-y(\boldsymbol{x}, t-\Delta t)}{2 \Delta t} .
$$

Then, we introduce the following fully discrete approximation of (27):

$$
\left\{\begin{array}{l}
\left(M_{1}\left(y^{n+1}\right)\right)_{j, k}=\left(M_{2}\left(y^{n}\right)\right)_{j, k}+\left(M_{3}\left(y^{n-1}\right)\right)_{j, k}, \forall(j, k) \in \boldsymbol{\Omega}_{\boldsymbol{h}_{\mathbf{1}}, \boldsymbol{h}_{\mathbf{2}}}, \forall n \in[0, N] \\
y_{j, k}^{n}=0, \quad \forall(j, k) \in \partial \boldsymbol{\Omega}_{\boldsymbol{h}_{\mathbf{1}}, \boldsymbol{h}_{\mathbf{2}}}, \quad \forall n \in[0, N]
\end{array}\right.
$$

with

and $r=\Delta t / h$. To initialize (89), we use

$$
\left\{\begin{array}{l}
\left(M_{1}(y)\right)_{j, k} \equiv\left(1+a_{j, k} \frac{\Delta t}{2}\right) y_{j, k}-\frac{\Delta t}{2}(M(y))_{j, k} \\
\left(M_{2}(y)\right)_{j, k} \equiv 2 y_{j, k}+r^{2}(M(y))_{j, k} \\
\left(M_{3}(y)\right)_{j, k} \equiv\left(-1+a_{j, k} \frac{\Delta t}{2}\right) y_{j, k}-\frac{\Delta t}{2}(M(y))_{j, k} \\
(M(y))_{j, k}=y_{j+1, k}+y_{j-1 k}+y_{j, k+1}+y_{j k-1}-4 y_{j, k}
\end{array}\right.
$$

$$
y_{j, k}^{0}=y_{0}\left(x_{1, j}, x_{2, k}\right), \quad \frac{y_{j, k}^{1}-y_{j, k}^{-1}}{2 \Delta t}=y_{1}\left(x_{1, j}, x_{2, k}\right), \quad \forall(j, k) \in \partial \boldsymbol{\Omega}_{\boldsymbol{h}_{\mathbf{1}}, \boldsymbol{h}_{\mathbf{2}}} .
$$

Writing the first relation of (89) for $n=0$, we obtain that the approximation of the solution $y$ at time $\Delta t$ is

$$
y_{j, k}^{1}=\frac{1}{2}\left(M_{2}\left(y_{0}\left(x_{1, j}, x_{2, k}\right)\right)-2 \Delta t M_{3}\left(y_{1}\left(x_{1, j}, x_{2, k}\right)\right)\right) .
$$

\section{Remark 4.1.}

- In the absence of damping and viscous terms, the scheme above reduces to $\left(M_{1}(y)\right)_{j, k}=\left(M_{3}(y)\right)_{j, k}=$ $y_{j, k}$ which coincides with the classical finite difference (explicit) scheme for the wave equation.

- Note that $M_{1}$ is not a diagonal matrix because of the presence of the viscous damping term. Therefore, the scheme (89) is implicit. 
Proposition 4.2. The implicit scheme (89) is consistent of order 2 in time and space and is stable under the CFL condition $\Delta t \leq h / \sqrt{2}$. Therefore, the scheme is convergent.

Proof. The first assertion is a direct consequence of (88). Concerning the stability condition, let us assume that the function $a(\boldsymbol{x}) \equiv a$ is constant in $\Omega$ and then perform a Von Neumann analysis. We assume that $y_{j, k}^{n}=\hat{y}^{n} \mathrm{e}^{i\left(j j_{x_{1}}+k k_{x_{2}}\right) \pi h}$, with $j_{x_{1}} \in[1, J], k_{x_{2}} \in[1, K]$ and replace this expression in (89). We obtain the following relation

with

$$
\hat{y}^{n+1}\left(1+C \frac{\Delta t}{2}\right)+\hat{y}^{n}\left(r^{2} \xi^{2}-2\right)+\hat{y}^{n-1}\left(1-C \frac{\Delta t}{2}\right)=0
$$

$$
C \equiv a+\xi^{2} ; \quad \xi^{2} \equiv 4\left(\sin ^{2}\left(k_{x_{1}} \pi h / 2\right)+\sin ^{2}\left(j_{x_{2}} \pi h / 2\right)\right) .
$$

According to the Von Neumann analysis, the scheme (89) is stable if the modulus of each root of the following polynomial is less than one;

$$
X^{2}\left(1+C \frac{\Delta t}{2}\right)+X\left(r^{2} \xi^{2}-2\right)+\left(1-C \frac{\Delta t}{2}\right)=0
$$

This is the case if

$$
2 r^{2} \xi^{2}\left(-4+r^{2} \xi^{2}\right)-4\left(a+\xi^{2}\right) \Delta t \leq 0 .
$$

Since $a \geq 0$, this inequality holds if $r^{2} \xi^{2} \leq 4$, i.e., if $r^{2} \leq 1 / 2$ which leads to the condition $\Delta t \leq h / \sqrt{2}$. From the Lax theorem (see [5]), we conclude that the scheme (89) is convergent.

\section{Remark 4.3.}

- The case where $a(\boldsymbol{x})$ is non constant can be treated using an energy method [5]. This leads to the same CFL condition.

- Using the approximation $\frac{\partial y}{\partial t}(\boldsymbol{x}, t) \approx(y(\boldsymbol{x}, t)-y(\boldsymbol{x}, t-\Delta t)) / \Delta t$ instead of (88) leads to the following explicit scheme:

with

$$
\left\{\begin{array}{l}
y_{j, k}^{n+1}=\left(M_{2}\left(y^{n}\right)\right)_{j, k}+\left(M_{3}\left(y^{n-1}\right)\right)_{j, k},(j, k) \in \boldsymbol{\Omega}_{\boldsymbol{h}_{\mathbf{1}}, \boldsymbol{h}_{\mathbf{2}}}, \forall n \in[0, N] \\
y_{j, k}^{n}=0, \forall(j, k) \in \partial \boldsymbol{\Omega}_{\boldsymbol{h}_{\mathbf{1}}, \boldsymbol{h}_{\mathbf{2}}}, \quad \forall n \in[0, N],
\end{array}\right.
$$

$$
\left\{\begin{array}{l}
\left(M_{2}(y)\right)_{j, k} \equiv\left(2-\Delta t a_{j, k}\right) y_{j, k}+\left(r^{2}+\Delta t\right)(M(y))_{j, k} \\
\left(M_{3}(y)\right)_{j, k} \equiv\left(-1+\Delta t a_{j, k}\right) y_{j, k}-(M(y))_{j, k}
\end{array}\right.
$$

and (92) being replaced by

$$
y_{j, k}^{1}=y_{0}\left(x_{1, j}, x_{2, k}\right)+\Delta t y_{1}\left(x_{1, j}, x_{2, k}\right) .
$$

However, this scheme is only of order one in time and is stable under a more restrictive CFL condition depending on $a$.

\subsection{Spectrum of the discrete damped wave operator}

In this section, we study the influence of the viscous terms on the spectrum of the discrete damped wave operator

$$
\mathcal{A}_{h}^{(p)}=\left(\begin{array}{cc}
0 & \boldsymbol{I}_{\boldsymbol{h}, \boldsymbol{\Omega}} \\
\boldsymbol{\Delta}_{\boldsymbol{h}} & -a \boldsymbol{I}_{\boldsymbol{h}, \boldsymbol{\omega}}+p h^{2} \boldsymbol{\Delta}_{\boldsymbol{h}}
\end{array}\right)
$$

We designate by $\sigma\left(\mathcal{A}_{h}^{(p)}\right)$ this spectrum and we recall that - at the continuous level - the exponential decay rate verifies the inequality: $\alpha \leq-\sup \{\operatorname{Re}(\lambda), \lambda \in \sigma(\mathcal{A})\}$. When $p=1$, the viscous terms are used. We consider the geometry of the Figure 3 included in the unit box (see also Fig. 4 ). The domain $\omega$ is represented by Figure 4. 

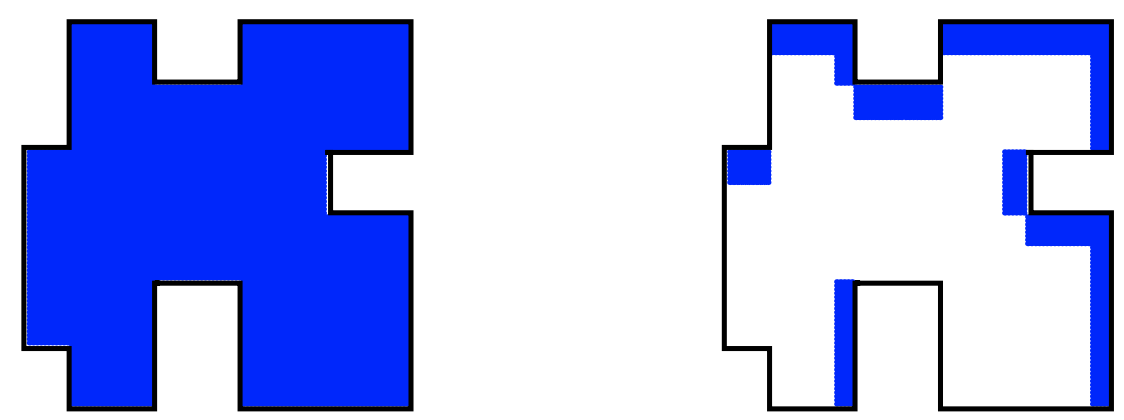

Figure 4. Domain $\Omega \subset(0,1)^{2}$ (left) and support $\omega \subset \Omega$ (right) of the damping function $a$ (right).
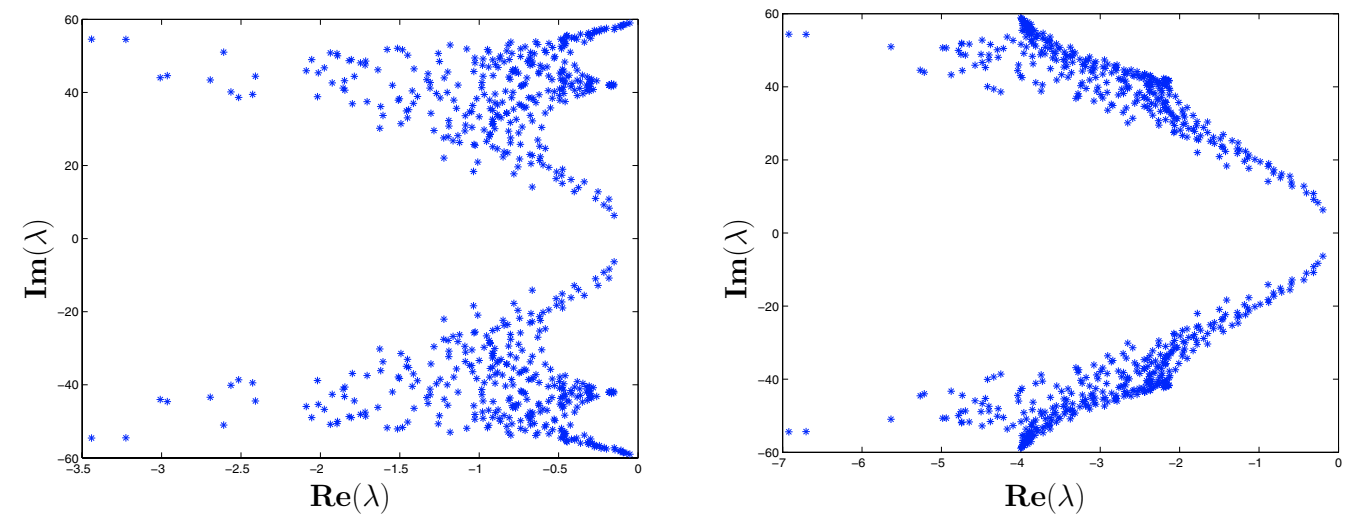

FIGURE 5. Location of the eigenvalues of $\mathcal{A}_{h}^{(\mathbf{0})}$ (left - without viscosity terms) and $\mathcal{A}_{h}^{(\mathbf{1})}$ (right - with viscosity terms).

TABLE 2. $-\max \left\{\operatorname{Re}(\lambda), \lambda \in \sigma\left(\mathcal{A}_{h}^{(p)}\right)\right\}$ without $(p=0)$ and with $(p=1)$ viscosity terms.

\begin{tabular}{c|cccc}
\hline & $h=1 / 20$ & $h=1 / 40$ & $h=1 / 80$ & $h=1 / 160$ \\
\hline without viscosity terms & 0.01532 & 0.00934 & 0.00312 & 0.00023 \\
\hline with viscosity terms & 0.2321 & 0.1924 & 0.1902 & 0.1898 \\
\hline
\end{tabular}

For $a(\boldsymbol{x})=10 \mathbb{1}_{\omega}(\boldsymbol{x})$ and $h=1 / 20$, Figure 5 depict the discrete spectrum $\sigma\left(\mathcal{A}_{\boldsymbol{h}}^{(\boldsymbol{p})}\right)$ for $p=0$ and $p=1$. As well-known, $\omega$ being fixed, the profile of the spectrum strongly depends on the function $a$. However, whatever $a$, we observe that without viscosity terms, the spectral abscissa $-\max \left\{\operatorname{Re}(\lambda), \lambda \in \sigma\left(\mathcal{A}_{h}^{(0)}\right)\right\}$ goes to zero with $h$. On the contrary, this quantity remains uniformly bounded by below when the viscosity terms are used (see Tab. 2). This clearly indicates the necessity of introducing viscosity term in order to restore the uniform exponential decay property. Moreover, in agreement with the Remark 2.2, we observe any difference on the spectral abscissa with and without viscosity term when the damping function is effective on the whole domain, i.e. when $\omega=\Omega$. We obtain $-\max \left\{\operatorname{Re}(\lambda), \lambda \in \sigma\left(\mathcal{A}_{h}^{(p)}\right)\right\} \geq 5$ for all $h>0$ and $p=0,1$. Clearly, in this case, whatever the speed of the wave packets of high frequencies, they reach the region $\omega$ in a finite time. Furthermore, we refer the reader to [1] for the computation of the spectrum for geometrically complex domain in $\mathbb{R}^{2}$. 


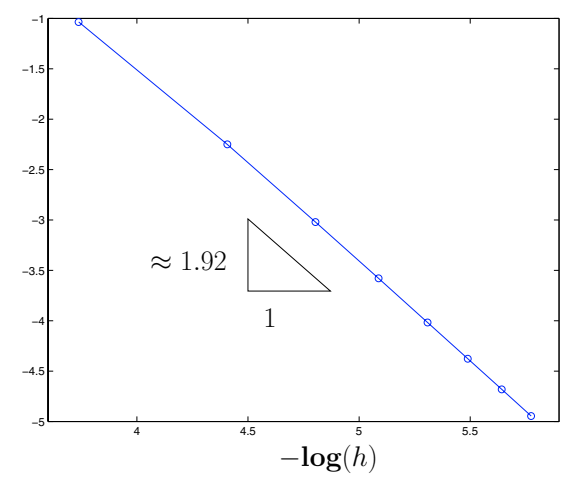

Figure $\quad 6 . \log \left(\mid E_{h}(0) \quad-\right.$ $\left.E_{\text {ref }}(0) \mid / E_{h}(0)\right)$ vs. $-\log (h)$.

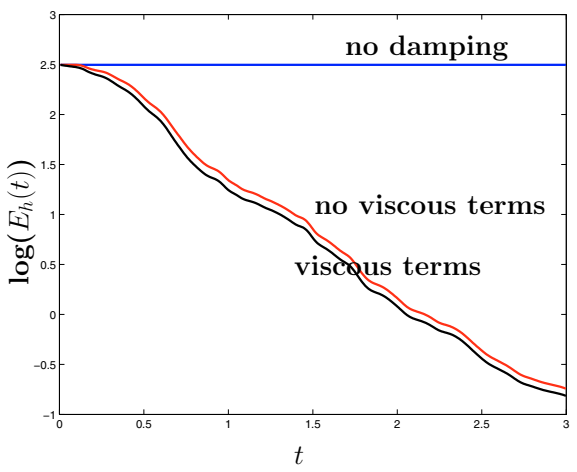

Figure 7. $\log \left(E_{h}(t)\right)$ vs. $t$, $h=1 / 100$.

TABLE 3. Exponential decay rate observed in the time interval $[0,3]$ vs. $h$.

\begin{tabular}{c|cccc}
\hline & $h=1 / 50$ & $h=1 / 100$ & $h=1 / 150$ & $h=1 / 200$ \\
\hline without viscosity terms & -1.16484 & -1.19264 & -1.19521 & -1.19655 \\
\hline with viscosity terms & -1.23830 & -1.20136 & -1.19892 & -1.19723 \\
\hline
\end{tabular}

\subsection{Numerical examples}

In this section, we present some numerical experiments for three different initial conditions. The first one concerns the simplest regular initial condition involving only the first component of the frequency mode (see Eq. (101)). The second one is a very singular one involving a discontinuous initial condition $y_{0}$. For these two cases, we consider the domain $\Omega$ - part of the unit square - described by Figure 4-left. Finally, we take $a(\boldsymbol{x})=10 \mathbb{1}_{\omega}(\boldsymbol{x})$ where $\omega \subset \Omega$ is described by Figure 4-right. The third example concerns a very oscillating function $y_{0}$ defined on the unit square.

\subsubsection{Regular initial conditions}

Let us designate by $D$ the disc of center $(1 / 2,1 / 2)$ and radius $\sqrt{0.02}$. We check that $D \subset \Omega \backslash \omega$. We then define the following initial conditions in $H_{0}^{1}(\Omega) \times L^{2}(\Omega)$ :

$$
y_{0}\left(x_{1}, x_{2}\right)=100\left[0.02-\left(x_{1}-1 / 2\right)^{2}-\left(x_{2}-1 / 2\right)^{2}\right] \mathbb{1}_{D}\left(x_{1}, x_{2}\right), \quad y_{1}\left(x_{1}, x_{2}\right)=0 \text {. }
$$

On $\Omega$, the function $y_{0}$ is positive and continuous.

For this function and this geometry, we check - in the conservative case - the order 2 of the convergence of the scheme (89). Figure 6 depicts the relative error of the energy $\left|E_{h}(0)-E_{\text {ref }}(0)\right| / E_{\text {ref }}(0)$ with respect to $h$ (in a $\log$-log scale): we obtain $\left|E_{h}(0)-E_{r e f}(0)\right|=O\left(h^{1.92}\right)$. The value of reference $E_{\text {ref }}(0)$ is obtained with $h=1 / 500$. Figure 7 describes the evolution of the energy for the conservative case, the case with velocity damping and the one with both velocity damping and viscous term. The scheme clearly reproduces the expected properties: in the conservative case, $E_{h}$ is constant in time whereas in the other cases, the decay rate is exponential. In the damped case (without viscous term), the energy $E_{h}$ is initially constant during a small period: this period corresponds to the time needed for the wave to reach $\omega$. We also observe that the viscous term has a low impact on the decay rate. The regularity of the function $y_{0}$ implies that $h^{2} \Delta y$ is negligible with respect to the other terms in equation (27): when $h$ goes to zero, both decay rates converge to the same value (see Tab. 3). 

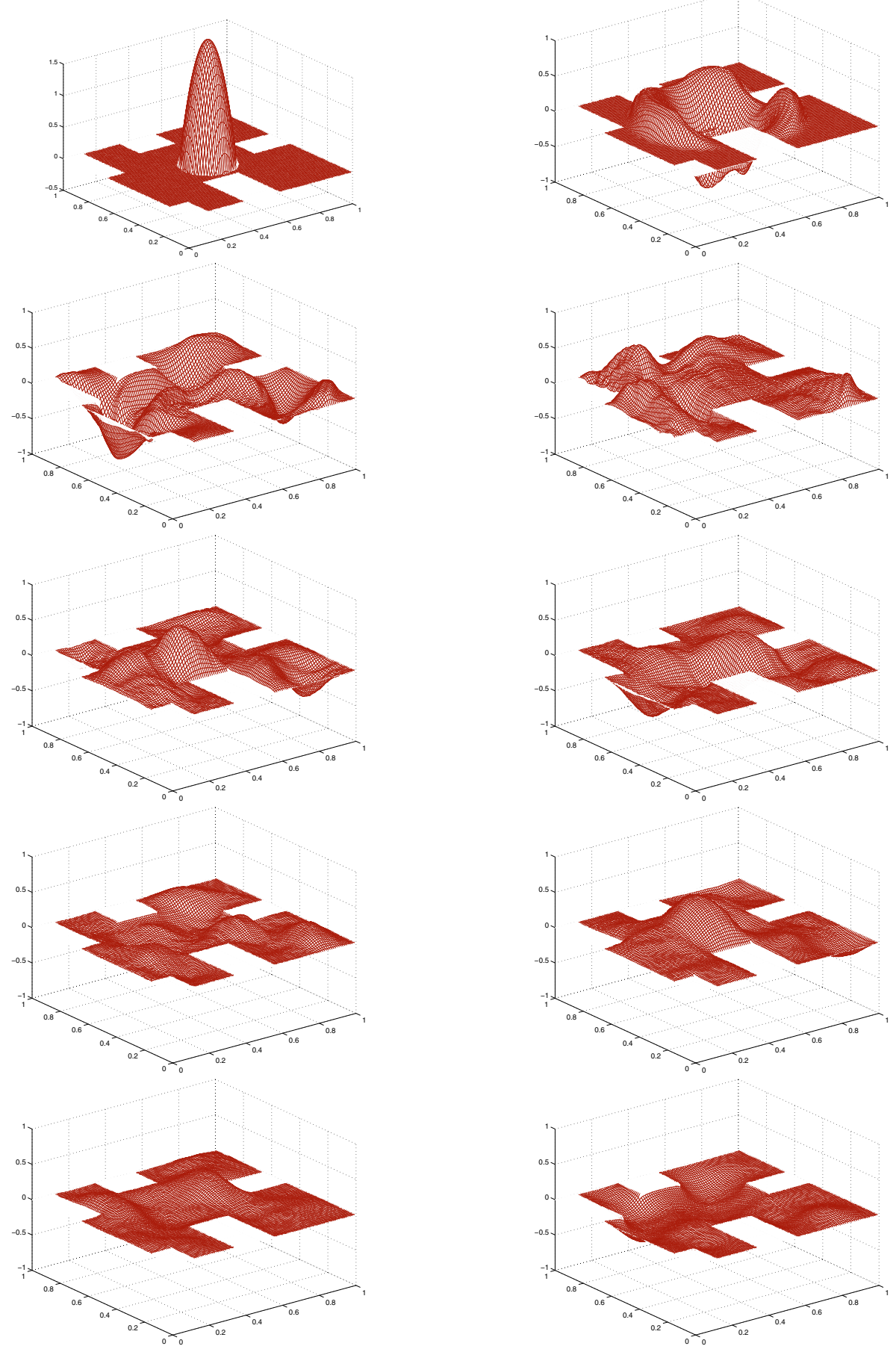

Figure 8. Solution $\boldsymbol{y}_{h}$ at time $t=0,1 / 4,2 / 4,3 / 4,4 / 4,5 / 4,6 / 4,7 / 4,8 / 4,9 / 4-E_{h}(0) \approx 11.98$, $E_{h}(1 / 4) \approx 11.38, E_{h}(2 / 4) \approx 8.83, E_{h}(3 / 4) \approx 5.56, E_{h}(4 / 4) \approx 3.95, E_{h}(5 / 4) \approx 3.18, E_{h}(6 / 4) \approx$ $2.41, E_{h}(7 / 4) \approx 1.72, E_{h}(8 / 4) \approx 1.21, E_{h}(9 / 4) \approx 0.95$. 
Finally, Figure 8 depicts the evolution of the solution for $t \in[0,9 / 4]$ obtained with $h=1 / 100$, highlighting the damping mechanism.

\subsubsection{Irregular initial conditions}

The previous section does not highlight the necessity of the viscous term. With the same geometry, we now consider the discontinuous initial conditions:

$$
y_{0}(\boldsymbol{x})=2 \mathbb{1}_{D}(\boldsymbol{x}) ; \quad y_{1}(\boldsymbol{x})=0 .
$$

In this case, the viscous term $h^{2} \Delta y$ is not negligible. Figures 9 and 10 represent the evolution of $E_{h}$ for $t \in[0,3]$ with and without extra viscous terms with mesh size $h=1 / 100$. In both cases, we observe an exponential decay rate. However, contrary to the first example, the decay rates are different (see Tab. 4). The viscous term permits to damped out the high frequencies components of the solution enhanced by the irregularity of $y_{0}$. Without viscous term, the time needed for all the wave packets to reach $\omega$ is larger (and dependent of $h$ ). After this time (large enough), the energy in both cases are similar. Finally, in order to appreciate the regularizing effect of the term $h^{2} \Delta y$, Figure 11 depicts in both cases the solution $\boldsymbol{y}_{\boldsymbol{h}}\left(x_{1}, 0.4\right)$ at time $t=2$.

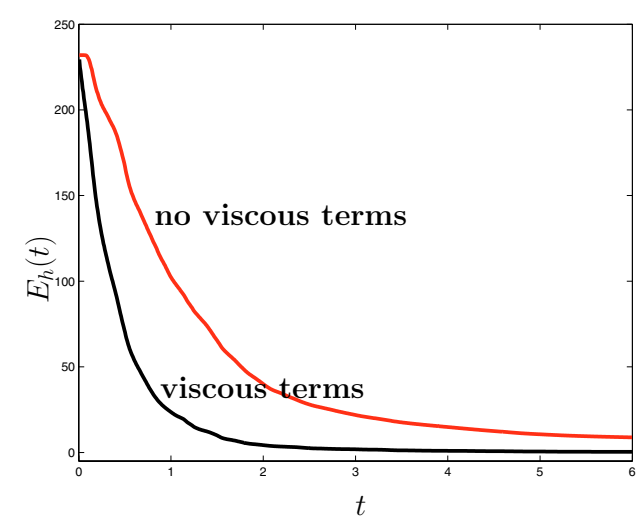

Figure 9. $E_{h}(t)$ vs. $t \in$ $[0,6], h=1 / 100$.

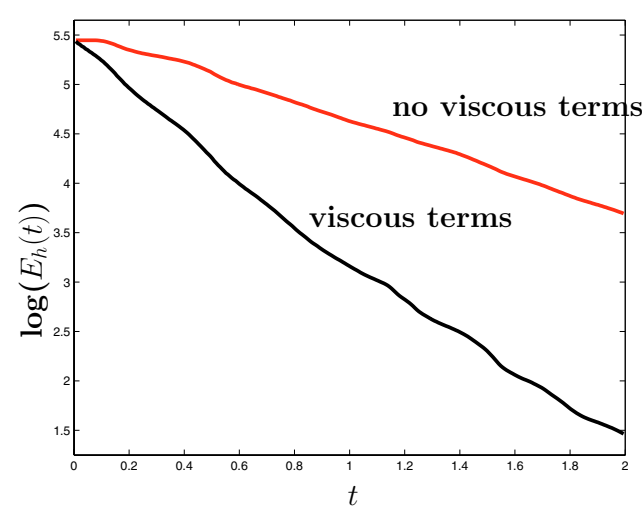

FiguRE $10 . \log \left(E_{h}(t)\right)$ vs. $t \in[0,2], h=1 / 100$.

TABLE 4. Exponential decay rate observed in the time interval $[0,2]$ vs. $h$.

\begin{tabular}{c|cccc}
\hline & $h=1 / 50$ & $h=1 / 100$ & $h=1 / 150$ & $h=1 / 200$ \\
\hline without viscosity terms & -0.94462 & -0.92221 & -0.90134 & -0.90012 \\
\hline with viscosity terms & -1.84121 & -2.00718 & -2.13322 & -2.155835 \\
\hline
\end{tabular}

\subsubsection{Influence of the high frequencies}

In the extreme case where the solution $y$ is only represented by high frequencies components, the term $h^{2} \Delta y$ becomes preponderant. On the unit square, let us now consider the very oscillating initial condition (but still continuous):

$$
y_{0}(\boldsymbol{x})=\sin \left(-\pi x_{1} \frac{1}{h}\right) \sin \left(-\pi x_{2} \frac{1}{h}\right)-\sin \left(\pi x_{1}\left(1-\frac{1}{h}\right)\right) \sin \left(\pi x_{2}\left(1-\frac{1}{h}\right)\right) ; \quad y_{1}(\boldsymbol{x})=0
$$




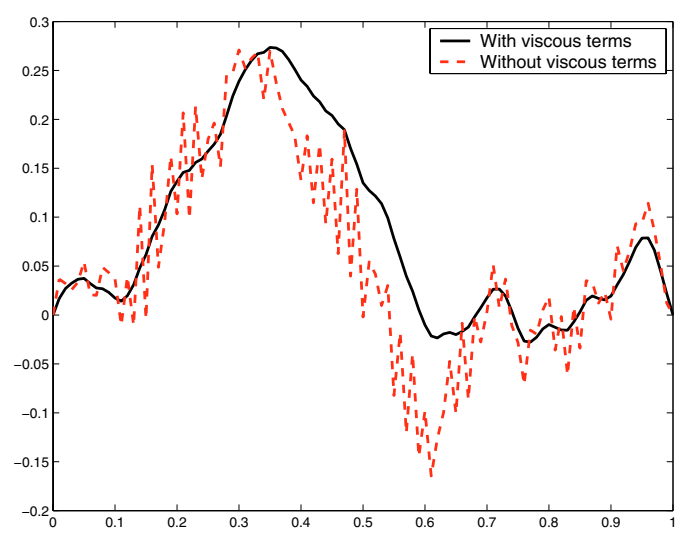

FiguRE 11. $\boldsymbol{y}_{\boldsymbol{h}}\left(x_{1}, 0.4, t=2\right)$ vs. $x_{1} \in[0,1]$ for $h=1 / 100$ with and without extra viscous terms.

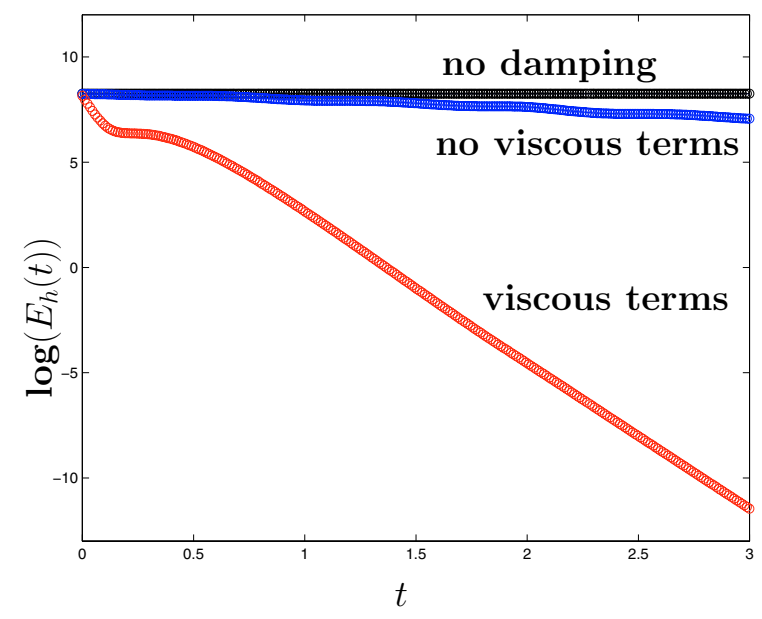

Figure 12. $\log \left(E_{h}(t)\right)$ vs. $t ; h=1 / 60$.

TABLE 5. Exponential decay rate observed in the time interval $[0,2]$ vs. $h$.

\begin{tabular}{c|ccccc}
\hline & $h=1 / 20$ & $h=1 / 40$ & $h=1 / 60$ & $h=1 / 80$ & $h=1 / 100$ \\
\hline without viscosity terms & -0.4256 & -0.4042 & -0.3911 & -0.3815 & -0.3700 \\
\hline with viscosity terms & -5.8714 & -6.4738 & -6.7190 & -6.8379 & -6.9098 \\
\hline
\end{tabular}

and $a(\boldsymbol{x}) \equiv 20 \mathbb{1}_{\omega}(\boldsymbol{x})$ where $\omega \equiv\left\{x_{1} \in[0.9,1], x_{2} \in[0,1]\right\} \cup\left\{x_{1} \in[0,1], x_{2} \in[0.9,1]\right\}$. These initial conditions lead to wave packets with group velocity of the order of the mesh size $h$ and they are the main obstacle for the uniform stabilization property to hold. We observe a clear difference on the behavior of the solution $\boldsymbol{y}_{\boldsymbol{h}}$ obtained with or without viscous damping. Without extra viscous terms, the velocity damping has no effect: computations lead to a rate of decay slowly converging toward zero when $h$ goes to zero. On the contrary, when the viscous term is added, we obtain a rate of decay near -7 (see Fig. 12 and Tab. 5). This is also illustrated in Figure 13 where we draw the evolution of $\boldsymbol{y}_{\boldsymbol{h}}\left(x_{1}, 1 / 2, t\right)$ for $t \in[0,2$.$] . Finally, it is worth mentioning that$ the use of the viscous scheme leads to a significant increase of the computational cost. 

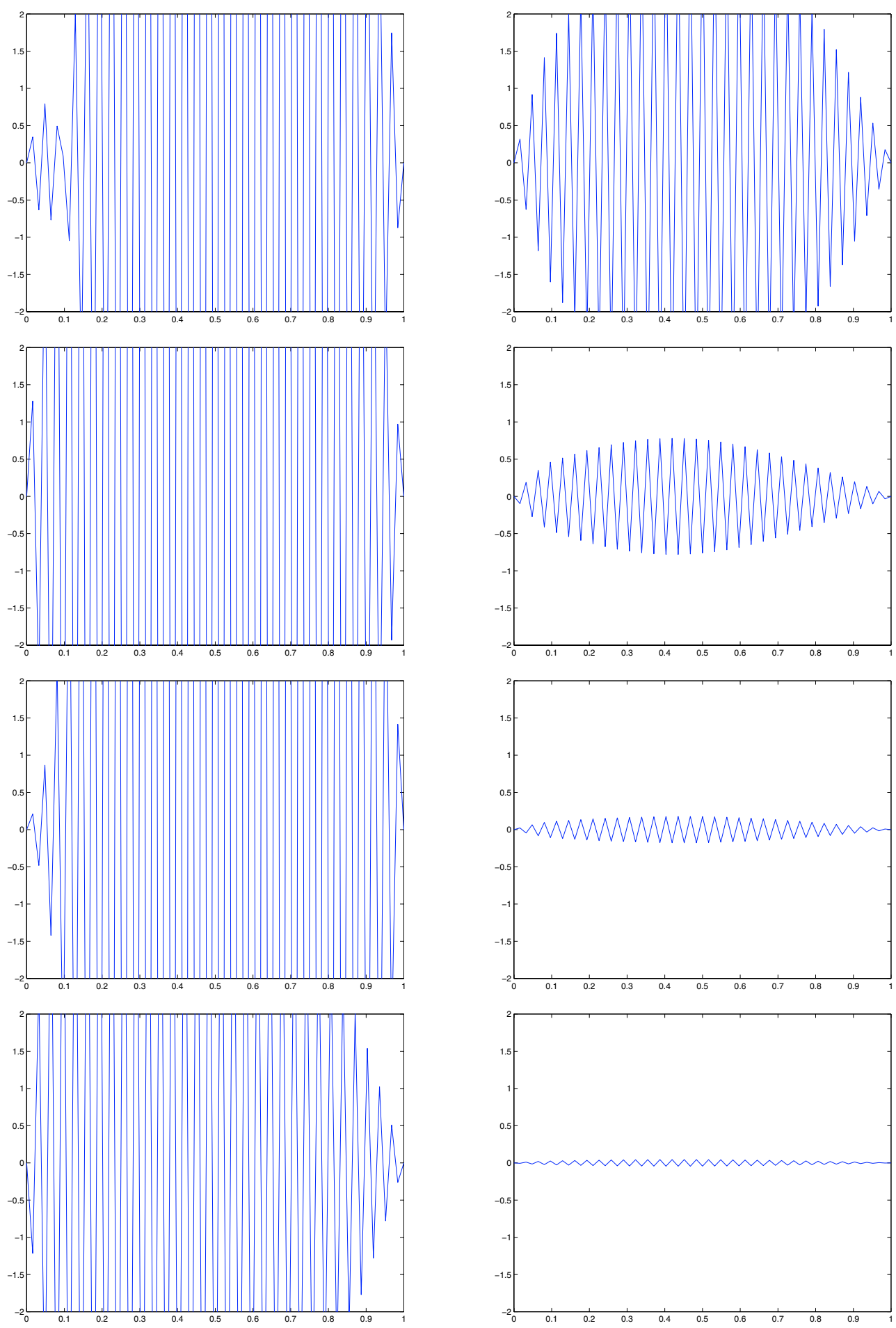

FiguRE 13. $\boldsymbol{y}_{\boldsymbol{h}}\left(x_{1}, 1 / 2, t\right)$ without (left) and with (right) extra viscous terms for $t=0.8,1.2,1.6,2$. 
Acknowledgements. We would like to thank the Referee of this journal for the constructive criticism which allow us to improve our earlier version. Professor Enrique Zuazua is also gratefully acknowledged for fruitful discussions and valuable comments on preliminary version of this work.

\section{REFERENCES}

[1] M. Asch and G. Lebeau, The spectrum of the damped wave operator for geometrically complex domain in $\mathbb{R}^{2}$. Experimental Math. 12 (2003) 227-241.

[2] H.T. Banks, K. Ito and B. Wang, Exponentially stable approximations of weakly damped wave equations. Ser. Num. Math. 100 Birkhäuser (1990) 1-33.

[3] C. Bardos, G. Lebeau and J. Rauch, Sharp sufficient conditions for the observation, control and stabilization from the boundary. SIAM J. Control Opt. 30 (1992) 1024-1065.

[4] D. Chenais, On the existence of a solution in a domain identification problem. J. Math. Anal. Appl. 52 (1975) $189-219$.

[5] G.C. Cohen, Higher-order Numerical Methods for Transient Wave Equations. Scientific Computation, Springer (2002).

[6] C.M. Dafermos, On the existence and the asymptotic stability of solutions to the equations of linear thermoelasticity. Arch. Rational Mech. Anal. 29 (1968) 241-271.

[7] R. Glowinski, C.H. Li and J.-L. Lions, A numerical approach to the exact boundary controllability of the wave equation (I). Dirichlet Controls: Description of the numerical methods. Japan. J. Appl. Math. 7 (1990) 1-76.

[8] A. Haraux, Stabilization of trajectories for some weakly damped hyperbolic equations. J. Differential Equations 59 (1985) $145-154$.

[9] A. Haraux, Une remarque sur la stabilisation de certains systèmes du deuxième ordre en temps. Portug. Math. 46 (1989) $245-258$.

[10] A. Henrot, Continuity with respect to the domain for the Laplacian: a survey. Control Cybernetics 23 (1994) $427-443$.

[11] J.A. Infante and E. Zuazua, Boundary observability for the space-discretizations of the 1-D wave equation. ESAIM: M2AN 33 (1999) 407-438.

[12] V. Komornik, Exact Controllability and Stabilization - The Multiplier Method. J. Wiley and Masson (1994).

[13] S. Krenk, Dispersion-corrected explicit integration of the wave equation. Comput. Methods Appl. Mech. Engrg. 191 (2001) 975-987.

[14] J. Lagnese, Control of wave processes with distributed control supported on a subregion. SIAM J. Control Opt. 21 (1983) 68-85.

[15] J.L. Lions, Quelques méthodes de résolution des problèmes aux limites non linéaires. Dunod-Gauthier-Villars, Paris (1969).

[16] J.L. Lions and E. Magenes, Problèmes aux limites non homogènes et applications, Vol. 1. Dunod, Paris (1968).

[17] A. Münch, A uniformly controllable and implicit scheme for the 1-D wave equation. ESAIM: M2AN 39 (2005) $377-418$.

[18] M. Nakao, Decay of solutions of the wave equation with a local degenerate dissipation. Israel J. Math. 95 (1996) $25-42$.

[19] M. Negreanu and E. Zuazua, Discrete Ingham inequalities and applications. C.R. Acad. Sci. Paris 338 (2004) $281-286$.

[20] O. Pironneau, Optimal shape design for elliptic systems. New York, Springer (1984).

[21] K. Ramdani, T. Takahashi and M. Tucsnak, Uniformly exponentially stable approximations for a class of second order evolution equations: Application to the optimal controle of flexible structures. Technical report, Prépublications de l'Institut Elie Cartan 27 (2003).

[22] M. Slemrod, Weak asymptotic decay via a "Relaxed Invariance Principle" for a wave equation with nonlinear, nonmonotone damping. Proc. Royal Soc. Edinburgh 113 (1989) 87-97.

[23] L.R. Tcheugoué-Tébou, Stabilization of the wave equation with localized nonlinear damping. J. Differential Equations 145 (1998) 502-524.

[24] L.R. Tcheugoué-Tébou and E. Zuazua, Uniform exponential long time decay for the space semi-discretization of a locally damped wave equation via an artificial numerical viscosity. Numer. Math. 95 (2003) 563-598.

[25] E. Zuazua, Exponential decay for the semilinear wave equation with locally distributed damping. Comm. Partial Differential Equation 15 (1990) 205-235.

[26] E. Zuazua, Boundary observability for finite-difference space semi-discretizations of the 2-D wave equation in the square. $J$. Math. Pures Appl. 78 (1999) 523-563.

[27] E. Zuazua, Optimal and approximate control of finite-difference approximation schemes for the 1-D wave equation. Rendiconti di Matematica, Serie VIII 24 (2004) 201-237.

[28] E. Zuazua, Propagation, observation, control and numerical approximation of waves. SIAM Rev. 47 (2005) 197-243. 